\title{
Non-singular black holes and the Limiting Curvature Mechanism: A Hamiltonian perspective
}

\author{
J. Ben Achour, ${ }^{a}$ F. Lamy, ${ }^{b}$ H. Liu ${ }^{b, c, d}$ and K. Noui ${ }^{b, d}$ \\ ${ }^{a}$ Center for Field Theory and Particles Physics, Fudan University \\ 200433 Shanghai, China \\ ${ }^{b}$ Laboratoire Astroparticule et Cosmologie, CNRS, Université Paris Diderot 7 \\ 75013 Paris, France \\ ${ }^{c}$ Centre de Physique Théorique (UMR CNRS 7332), Université d'Aix-Marseille et de Toulon \\ 13288 Marseille, France \\ ${ }^{d}$ Laboratoire de Mathématiques et Physique Théorique (UMR CNRS 7350), Université Fran- \\ cois Rabelais, Parc de Grandmont \\ 37200 Tours, France
}

E-mail: jibrilbenachour@gmail.com, lamyf@apc.in2p3.fr, liuh@apc.in2p3.fr, karim.noui@lmpt.univ-tours.fr

\begin{abstract}
We revisit the non-singular black hole solution in (extended) mimetic gravity with a limiting curvature from a Hamiltonian point of view. We introduce a parameterization of the phase space which allows us to describe fully the Hamiltonian structure of the theory. We write down the equations of motion that we solve in the regime deep inside the black hole, and we recover that the black hole has no singularity, due to the limiting curvature mechanism. Then, we study the relation between such black holes and effective polymer black holes which have been introduced in the context of loop quantum gravity. As expected, contrary to what happens in the cosmological sector, mimetic gravity with a limiting curvature fails to reproduce the usual effective dynamics of spherically symmetric loop quantum gravity which are generically not covariant. Nonetheless, we exhibit a theory in the class of extended mimetic gravity whose dynamics reproduces the general shape of the effective corrections of spherically symmetric polymer models, but in an undeformed covariant manner. These covariant effective corrections are found to be always metric dependent, i.e. within the $\bar{\mu}-$ scheme, underlying the importance of this ingredient for inhomogeneous polymer models. In that respect, extended mimetic gravity can be viewed as an effective covariant theory which naturally implements a covariant notion of point wise holonomy-like corrections. The difference between the mimetic and polymer Hamiltonian formulations provides us with a guide to understand the deformation of covariance in inhomogeneous polymer models.
\end{abstract}

\footnotetext{
${ }^{1}$ Corresponding author.
} 


\section{Contents}

1 Introduction $\quad \mathbf{2}$

1.1 Motivations: mimetic gravity as a guide to derive a covariant effective loop regularization

1.2 Equivalent formulations and relation to DHOST theories 3

1.3 Limiting curvature hypothesis 4

1.4 Towards an effective description of polymer black hole in Loop Quantum Gravity? 4

1.5 On the effective description of quantum black holes in LQG 6

1.6 Layout of the paper $\quad 7$

2 Mimetic gravity and the limiting curvature hypothesis $\quad 8$

2.1 Extended mimetic gravity 8

2.1.1 Equations of motion 8

2.1.2 Generalization: extended mimetic gravity 9

$\begin{array}{llr}2.2 & \text { Black hole with a limiting curvature } & 10\end{array}$

3 Hamiltonian description $\quad 12$

$\begin{array}{lll}3.1 & 3+1 \text { decomposition: metric and scalar field } & 12\end{array}$

$\begin{array}{lll}3.1 .1 & \text { ADM decomposition for spherical space-time } & 12\end{array}$

3.1.2 Resolution of the mimetic condition to integrate out the scalar field 12

3.2 Hamiltonian analysis 13

3.3 Spherically "static" solutions deeply inside the black hole 14

$\begin{array}{ll}\text { 3.3.1 Equations of motion } & 15\end{array}$

$\begin{array}{ll}\text { 3.3.2 Deep inside the black hole: resolution of the singularity } & 15\end{array}$

$\begin{array}{ll}\text { 3.3.3 Comparing with the Schwarzschild solution } & 16\end{array}$

4 On the relation with effective polymer black holes $\quad 17$

$\begin{array}{lll}4.1 & \text { Spherically symmetric polymer models: a short review } & 17\end{array}$

4.1.1 The classical vacuum spherically symmetric Ashtekar-Barbero phase space

$\begin{array}{lll}\text { 4.1.2 The holonomy corrected polymer phase space } & 18\end{array}$

$\begin{array}{ll}\text { 4.1.3 Comparing mimetic and polymer black holes } & 20\end{array}$

4.2 Polymer Hamiltonians from extended mimetic gravity 22

$\begin{array}{lll}\text { 4.2.1 General construction } & 22\end{array}$

4.2.2 A first example: the standard anomaly free sine function corrections $\quad 24$

4.2.3 A second example: the Tibrewala's effective corrections 25

5 Conclusion 


\section{Introduction}

\subsection{Motivations: mimetic gravity as a guide to derive a covariant effective loop regularization}

Effective symmetry reduced polymer models of quantum geometry provide us with an interesting framework to investigate the deviations from General Relativity induced by the quantum nature of the geometry. These models are inspired by Loop Quantum Gravity, from which the regularization and quantization technics are borrowed. In its canonical form, the regularization step is performed at the level of the phase space, prior to quantization, such that the canonical variables of GR as well as the first class constraints encoding the gauge symmetry are modified. From this perspective, two natural questions appear regarding the consistency of the approach:

- i) First, does the classical regularization generate anomalies or deformations in the algebra of first class constraints, i.e. in the Dirac's hypersurface deformation algebra (DHDA) ?

- ii) Second, can one obtain an effective covariant action which reproduces, in the symmetry reduced sector under consideration, the effective quantum corrections implemented at the hamiltonian level ?

Naturally, these two questions are intimately related since no covariant action could reproduce effective hamiltonians corrections if they generate deformation of the Dirac's algebra, as it is the case in most inhomogeneous polymer models [1-7].

In the homogeneous cosmological sector, where there is no spatial diffeomorphism, the question i) of anomaly freedom naturally disappears and the DHDA is trivial. Therefore the regularization is not constrained by any anomaly free conditions. One can thus derive en effective cosmological phase space by introducing the effective corrections inspired by LQG, and study the modified system. The resulting effective quantum cosmology, i.e. Loop Quantum Cosmology, describes a bouncing universe with a consistent semi-classical limit. One can quantize this system using again technics from LQG and show that for sufficiently semi-classical states, the quantum dynamics coincides with the effective classical dynamics, reinforcing the consistency of the approach at least in this cosmological sector. Moreover, the effective dynamics of LQC can be derived from a covariant scalar tensor lagrangian belonging to the extended mimetic gravity family $[8,9]$. Hence, in this cosmological case, question ii) can be answered in the affirmative.

A natural question is whether the successful construction of LQC, implementing the (partial) loop regularization with its associated effective (mimetic) action, can be generalized to inhomogeneous gravitational systems. For example, does it extend to the inhomogeneous spherically symmetric case? Does the solution of a regular black hole interior found in [10] (from the Chamesddine-Mukhanov's lagrangian which reproduces the effective dynamics of LQC in the cosmological sector) is related to the effective polymer black hole models $[1,11-13]$ studied so far?

As explained in more details in Sec.I.D, a first obstacle in generalizing the construction of LQC and its relation the extended mimetic theory found in $[8,9]$, is that when going to inhomogeneous situations, such as inhomogeneous cosmology, spherical symmetry as well as Gowdy system, point i) is no more satisfied. When no local degrees of freedom are involved, the Dirac's algebra has been shown to be deformed by holonomy corrections, while in presence 
of local degrees of freedom, the DHDA is anomalous. These results call therefore for a generalization of the current loop regularization used in inhomogeneous polymer models. Yet, as it stands, the current situation prevents from the start to derive an covariant effective action for these inhomogeneous models as done for homogeneous LQC in [8, 9].

In this work, we adopt to following strategy: we assume that extended mimetic gravity can indeed provide a consistent effective theory of polymer quantum gravity and study its symmetry reduction to spherical geometry. We propose a general procedure which, given some holonomy corrections, allow to derive the associated effective action belonging to the extended mimetic gravity family. This will provide a guide in investigating the issue of covariance in inhomogeneous polymer black hole models.

Having presented our motivations, let us now review briefly the extended mimetic gravity framework and the limiting curvature mechanism which will be a corner stone of our work. In order to be self complete, we also include a brief review of the recent results concerning the above mentioned issue of covariance in inhomogeneous effective polymer models. In the following, we shall use Planck units, i.e $G=c=1$.

\subsection{Equivalent formulations and relation to DHOST theories}

Mimetic gravity [15] is a theory of modified gravity which belongs to the family of scalar-tensor theories. There have been numerous generalizations of mimetic gravity, but the simplest (and original) theory is simply defined by the Einstein-Hilbert action for a metric $\tilde{g}_{\mu \nu}$

$$
\tilde{S}\left[\tilde{g}_{\mu \nu}, \phi\right]=\int d^{4} x \sqrt{-g} \mathcal{R} \quad \text { where } \quad g_{\mu \nu} \equiv-\tilde{X} \tilde{g}_{\mu \nu} \quad \text { and } \quad \tilde{X} \equiv \tilde{g}^{\mu \nu} \phi_{\mu} \phi_{\nu} .
$$

where we use the standard notation $\phi_{\mu}=\partial_{\mu} \phi$ and $X=\phi^{\mu} \phi_{\mu}$. The metrics $g_{\mu \nu}$ and $\tilde{g}_{\mu \nu}$ are related by a non-invertible conformal transformation: if one can uniquely define $g_{\mu \nu}$ from $\tilde{g}_{\mu \nu}$, the reverse is not true. Indeed, the metric $\tilde{g}_{\mu \nu}$ is obviously defined up to a conformal transformation only.

The Euler-Lagrange equations derived from this action (1.1) are rather different from the Einstein equations even if the action reduces to the Einstein-Hilbert action for $\tilde{g}_{\mu \nu}$. To make this distinction clear, let us remark that the metric $g_{\mu \nu}$ satisfies the constraint $X \equiv$ $g^{\mu \nu} \phi_{\mu} \phi_{\nu}=-1$, which allows us to reformulate the action (1.1) equivalently as

$$
S\left[g_{\mu \nu}, \phi, \lambda\right]=\int d^{4} x \sqrt{-g}[\mathcal{R}+\lambda(X+1)],
$$

where $\lambda$ is a new dynamical variable. It is easy to show that these two actions (1.1) and (1.2) produce two equivalent sets of equations of motion. Thus, mimetic gravity appears to be equivalent to gravity coupled to the scalar fields $\phi$ and $\lambda$. A careful analysis shows that the theory propagates only one scalar degree of freedom in addition to the usual two tensorial modes (see [16] but also [19] for a Hamiltonian analysis of general higher-order scalar-tensor theories). Notice that, in its first formulation (1.1), the theory is clearly conformally invariant: the metric $\tilde{g}_{\mu \nu}$ and thus the action itself is invariant under the transformation $g_{\mu \nu} \mapsto \Omega(x) g_{\mu \nu}$ where $\Omega$ is an arbitrary function on the space-time. Hence, the conformal mode of the metric becomes dynamical, and this is the deep reason why the theory propagates one more degree of freedom than general relativity. Since mimetic gravity has been introduced, it has been studied extensively mainly in the context of cosmology (see [17] and references therein).

Starting from the first formulation (1.1), one can easily expand the Ricci scalar $\mathcal{R}$ and the measure $\sqrt{-g}$ in terms of $\tilde{g}_{\mu \nu}$ and $\phi$, and show that the action contains higher derivatives 
of the scalar field according to

$$
\tilde{S}\left[\tilde{g}_{\mu \nu}, \phi\right]=-\int d^{4} x \sqrt{-\tilde{g}}\left[\tilde{X} \tilde{\mathcal{R}}+\frac{6}{\tilde{X}} \tilde{g}^{\mu \rho} \tilde{g}^{\nu \alpha} \tilde{g}^{\sigma \beta} \phi_{\mu \nu} \phi_{\rho \sigma} \phi_{\alpha} \phi_{\beta}\right],
$$

where $\tilde{\mathcal{R}}$ is the Ricci scalar associated to the metric $\tilde{g}_{\mu \nu}$ and $\phi_{\mu \nu}=\nabla_{\mu} \partial_{\nu} \phi$. In that way, mimetic gravity can be viewed explicitly as a higher-order scalar-tensor theory which is moreover degenerate in the sense of [18]. Thus, it belongs to the family of Degenerate Higher-Order Scalar-Tensor (DHOST) theories [19-23], which confirms that it propagates (up to) only three degrees of freedom: one scalar and two tensorial modes. The fact that (1.3) is degenerate is closely related to the conformal invariance of the theory. This extra symmetry insures the presence of an extra (first class) constraint in the theory (in addition to the usual Hamiltonian and vectorial constraints) which makes the theory degenerate. Notice that the conformal invariance has been somehow broken in the second formulation of the theory (1.2) at the cost of bringing in the scenario an extra scalar degree of freedom $\lambda$.

\subsection{Limiting curvature hypothesis}

The initial physical motivation for considering the theory (1.1) has been to propose an alternative to cold dark matter in the universe. Actually, it reproduces exactly the results of a model introduced earlier by Mukohyama in [24]. Later on, the original proposal of [15] has been extended, in adding a potential $V(\phi)$ for the scalar field in (1.1) and has been shown to provide potentially interesting models for both the early universe and the late time cosmology [25]. Finally, more recently, mimetic gravity has been applied to construct non-singular cosmologies and non-singular black holes in $[10,26]$. Physically, the idea is very simple and consists in finding higher-order scalar-tensor Lagrangians (in the family of generalized mimetic gravity) in such a way that their Euler-Lagrange equations impose an upper limit on the Ricci scalar $\mathcal{R}$ : this is called the limiting curvature hypothesis. If this is the case, $\mathcal{R}$ never diverges and one could expect to resolve in that way some divergences which appear in classical general relativity.

Such a hypothesis can be implemented concretely and, in some situations, it is sufficient to transform the original cosmological singularity into a non-singular bounce in the context of homogeneous and isotropic space-times [26], and also to remove the black-hole singularity in the context of spherically symmetric space-times. The limiting curvature hypothesis was extended very recently in [27] to construct theories whose equations of motion impose upper limits not only on the Ricci scalar but also on any invariant constructed from the Riemann tensor $\mathcal{R}_{\mu \nu \rho \sigma}$ such as the Ricci tensor squared $\mathcal{R}_{\mu \nu} \mathcal{R}^{\mu \nu}$, the Weyl tensor squared $C^{2}=C_{\mu \nu \rho \sigma} C^{\mu \nu \rho \sigma}$, or invariants involving derivatives of the Riemann. However, as the authors pointed out in [27], these extended Lagrangians contain higher-order derivatives of the metric (which is not the case for the original mimetic gravity) and presumably suffer from Ostrogradski instabilities generically [28]. Moreover, it should be stress that up to now, no investigation on the stability of such models in the spherically symmetric framework has been performed. As a result, one should consider these theories of gravity with a limiting curvature as effective descriptions which are physically valuable up to some energy scale only.

\subsection{Towards an effective description of polymer black hole in Loop Quantum Gravity?}

Interestingly, as mentioned earlier, it was realized in $[8,9]$ that the original theory of mimetic gravity with a limiting curvature introduced in [26] reproduces exactly the effective Loop 
Quantum Cosmology bounce (LQC) [29] as a homogeneous and isotropic solution ${ }^{1}$. Naturally, such an observation raises the question of whether one could consider mimetic gravity with a limiting curvature as an effective description (up to some energy scale) of the full theory of Loop Quantum Gravity (LQG), even out of the cosmological sector.

Nonetheless, there are at least two obstacles for such a scenario to be possible. On the one hand, LQC is not a subsector of the full theory of LQG but rather a homogenous symmetry reduced model which is quantized importing the technics of the full LQG theory. Despite some preliminary results concerning the embedding of LQC into LQG [31-35], this aspect remains up to now poorly understood. Therefore the relation established between LQC and mimetic gravity does not say much a priori about an eventual link between LQG and mimetic gravity. On the other hand, even without considering the embedding of LQC into LQG, one could wonder whether the result obtained in $[8,9]$ can be generalized to less symmetric situations, such as spherically symmetric [11] or Gowdy loop models [36]. Such symmetry reduced models exhibit non perturbative inhomogeneities, and as such, are highly non trivial to quantize using the LQG technics. The reason is that, contrary to the homogenous cosmological sector, the invariance under spatial diffeomorphisms survives the symmetry reduction. Therefore, the loop quantization of such inhomogenous models faces the additional difficulty of keeping the spatial covariance (in the sense of the symmetry reduced model) in the quantization. More precisely, the issue of the covariance takes the form of requiring (at the quantum level) an anomaly free Dirac's hypersurface deformation algebra (DHDA), which is the algebra of the first class constraints generating the invariance under diffeomorphisms. Classically, this algebra is generated by the Hamiltonian constraint $\mathcal{H}$, the vectorial constraints $D_{a}$ satisfying

$$
\begin{aligned}
& \left\{D\left[U^{a}\right], D\left[V^{b}\right]\right\}=D\left[\mathcal{L}_{U} V^{a}\right], \quad\left\{D\left[U^{a}\right], \mathcal{H}[N]\right\}=-\mathcal{H}\left[\mathcal{L}_{U} N\right], \\
& \{\mathcal{H}[N], \mathcal{H}[M]\}=D\left[\gamma^{a b}\left(N \partial_{b} M-M \partial_{b} N\right)\right],
\end{aligned}
$$

where we have used the following standard notations: $U=U^{a} \partial_{a}$ and $V=V^{a} \partial_{a}$ are spatial vectors, $N$ and $M$ are scalars, $D\left[U^{a}\right]$ and $\mathcal{H}[N]$ are the smeared vectorial and Hamiltonian constraints respectively, $\gamma^{a b}$ is the inverse spatial metric and $\mathcal{L}_{U}$ denotes the usual Lie derivative along the vector $U$. In the quantum theory, this algebra is modified. Indeed, introducing point-wise holonomy corrections to regularize the first class constraints, as required by the loop quantization procedure, has very important consequences. The fate of the DHDA under the introduction of the holonomy corrections has been studied recently in a series of papers [1-5]. A generic result is that for system having no local degrees of freedom, such as vacuum spherically symmetric gravity [1], or Gowdy model with local rotational symmetry [2], the DHDA exhibits a deformed notion of covariance in the sense that (1.5) is replaced by

$$
\{\mathcal{H}[N], \mathcal{H}[M]\}=\beta(\tilde{K}) D\left[\gamma^{a b}\left(N \partial_{b} M-M \partial_{b} N\right)\right],
$$

where $\beta(\tilde{K})$ is a "deformation", called sometimes the "sign change deformation", which depends generically on the homogeneous component of the extrinsic curvature $\tilde{K}$. For systems having local degrees of freedom, the situation is even worse since the DHDA has been shown not to be a closed algebra anymore.

All this seems to lead us to the conclusion that it is not possible to provide an effective description of such loop quantized phase space using a covariant scalar tensor theory such

\footnotetext{
${ }^{1}$ Notice that a very similar construction has been considered much earlier in [30] in an attempt to reproduce the effective dynamics of LQC from an $f(R)$ theory.
} 
as [26, 27], since these theories share the same (undeformed) DHDA as classical General Relativity (augmented with a scalar degree of freedom). A first notorious complication in such model is that the quantum corrections are implemented at the Hamiltonian level and going back to a Lagrangian formulation is often very complicated (see [37] for earlier investigations on this aspect). Nonetheless, the situation is not totally hopeless. There are at least two reasons to think that one can circumvent these apparent obstacles we have discussed above. The first one relies on the observation that the difficulties related to the deformation of the DHDA seems to be inherent to the fact of working with the real Ashtekar-Barbero variables. Recent results have shown that such difficulties disappear when working with the initial selfdual variables, at least in the case of spherical symmetry gravity coupled to matter and unpolarized Gowdy models [38, 39]. In this situation, the DHDA keeps indeed its classical form without any deformation even if some holonomy corrections, including a $\bar{\mu}$-scheme, have been taken into account. Therefore, this opens the possibility of describing these self-dual loop symmetry reduced models using a covariant scalar-tensor theory. The second one relies on the fact that up to now, the polymer construction of black hole models (as well as inhomogeneous cosmology and Gowdy system) has focused only on the minimal loop regularization, namely the point-wise holonomy corrections. Yet, only few investigations have considered together the additional corrections inherent to LQG: the triad corrections related to the inverse volume regularization, as well as the holonomy of the inhomogeneous component of the connection. Therefore, it is not clear if the deformation (1.6) will not be removed when one takes into account these additional corrections.

Facing the problem of the deformed notion of covariance in polymer models inspired from LQG, we adopt the following strategy. As shown in [8, 9], mimetic gravity with a limiting curvature is a covariant scalar-tensor theory which reproduces in the cosmolgical sector the effective dynamics of LQC. Thus, we consider this theory as a potential guide to obtain an effective Lagrangian description of point wise holonomy-like corrections which maintain the covariance beyond the cosmological sector. As a first study, we focus our attention on the spherically symmetric sector and derive its Hamiltonian formulation. As expected, it leads to effective quantum corrections similar to the ones introduced in the polymer framework, but in a covariant manner. The difference between the mimetic effective corrections and the polymer ones provides an interesting guide to understand the lack of covariance of polymer black hole models.

\subsection{On the effective description of quantum black holes in LQG}

Before closing this introduction, let us explain more precisely how quantum black holes have been treated in the framework of LQG. The first descriptions of quantum black holes in LQG were introduced in $[40,41]$. They allowed to obtain a complete description of the black hole microstates whose counting is very well-known to reproduce the Bekenstein-Hawking formula at the semi-classical limit. Even though important issues concerning the role of the BarberoImmirzi parameter in the counting procedure remain unsolved, (see [42-49] for some proposals to overcome them), this result has been an important success for LQG. Unfortunately, these studies do not say much about the dynamical "geometry" of the quantum black holes in the context of LQG: microstates are described in terms of algebraic structures (intertwiners between representations) and recovering the geometrical content of the quantum black holes at the semi-classical limit is a very complicated task which required coarse-graining schemes still under development. One way to attack the problem would be to find, exactly as it was 
done in LQC, an effective description of spherically symmetric solutions and see how quantum gravity effects modifies Einstein equations in this sector.

Such a program, which relies on a polymer quantization of spherically symmetric geometries, was initiated in [51] and further developed in [52-55]. The effective dynamics of the interior black hole was explored in [56] and its construction follows very closely what is done in LQC, the black hole interior geometry being a homogenous anisotropic cosmology. However, the treatment of the whole space-time and its inhomogenous exterior geometry remains more challenging. While the loop quantization of the inhomogeneous vacuum exterior geometry was worked out in details in [11,57], an effective theory of this quantum geometry is not yet available (see [57] for a review on the quantization of the full spherically symmetric geometry). Moreover, going beyond the vacuum case and including matter has also proved to be very challenging, mainly because the standard holonomy corrections spoil generally the covariance of such models [1,4]. For this reason, studying an effective inhomogenous gravitational collapse including the quantum corrections from loop quantum gravity is up to now out of reach with the standard techniques. Another way to understand the geometry of quantum black holes (at the semi-classical limit) would be to "guess" the modifications induced by quantum gravity effects in mimicking LQC as it was done in [59] where the notion of "Planck stars" has been introduced. Some phenomenological aspects of these potentially new astrophysical objects have been studied in [59-62]. While the heuristic idea of the Planck stars is fascinating, a quantum theory of spherically symmetric geometry coupled to matter from which such effective description emerged still has to be built.

Here, we investigate an alternative way to study the issue of covariance in polymer models inspired by LQG using mimetic gravity. We start with a Hamiltonian analysis of the theory [26] and we revisit the non-singular black hole solution from a Hamiltonian point of view. In particular, we find the expression of the Hamiltonian constraint which could be interpreted as an effective Hamiltonian constraint for spherically symmetric geometries in quantum gravity. However, this expression differs from the ones that have been considered so far in the literature (even though their classical limits obviously agree). Nonetheless, this offers a new regularization scheme to be compared with the one obtained in standard spherically symmetric loop models [57]. One outcome of our approach is that the effective holonomy-like corrections have a natural inbuilt $\bar{\mu}$-scheme, contrary to the holonomy corrections usually introduced in the standard loop treatment [57] (see [7] for an attempt to include phase space dependent holonomy corrections in spherically symmetric polymer models). Moreover, we show that one can easily modify the initial proposal of [26] in order to obtain a theory of mimetic gravity which reproduces the usual effective Hamiltonian constraint of LQG for spherically symmetric geometries, including again an additional $\bar{\mu}$-scheme.

\subsection{Layout of the paper}

The paper is organized as follows. In section II, we recall some important aspects of the extended theories of mimetic gravity and we illustrate the limiting curvature mechanism in the context of black holes. In section III, we revisit the non-singular black hole solution described in [26] from a Hamiltonian point of view. In particular, we find a parametrization of the phase space which makes the resolution of the equations of motion simpler than in the Lagrangian formulation. Furthermore, we exhibit the explicit form of the Hamiltonian constraint for mimetic gravity with a limiting curvature in the case of a spherically symmetric space-time. In section IV, we compare the Hamiltonian formulation of this theory of mimetic gravity with those obtained in LQG from a "loop" regularization of the usual Hamiltonian 
constraint of gravity. Contrary to what happens in the cosmological sector, the theory of mimetic gravity proposed in [26] does not reproduce the effective dynamics of spherically symmetric LQG. However, we exhibit a theory in the class of extended mimetic gravity whose dynamics reproduces the general shape of the effective corrections of spherically symmetric polymer models, but in an undeformed covariant manner. In that respect, extended mimetic gravity can be viewed as an effective covariant theory which naturally implements a covariant notion of point wise holonomy-like corrections similar in spirit to the ones used in polymer models. The difference between the mimetic and polymer Hamiltonian formulations provides us with a guide to understand the lack of covariance in inhomogeneous polymer models.

\section{Mimetic gravity and the limiting curvature hypothesis}

In this section, we review some aspects of extended theories of mimetic gravity [63, 64]. Extended mimetic gravity generalizes the original proposal of [15]: it is still conformally invariant and propagates generically only one scalar in addition to the usual two tensorial modes. Then, we recall how to implement the limiting curvature hypothesis in this context and how the proposal of [26] leads indeed to a non-singular black hole solution.

\section{$2.1 \quad$ Extended mimetic gravity}

Let us start by discussing the original theory of mimetic gravity defined by the action (1.2)

$$
S\left[g_{\mu \nu}, \phi, \lambda\right] \equiv \int d^{4} x \sqrt{-g}\left[\frac{1}{2} \mathcal{R}+\lambda\left(g^{\mu \nu} \phi_{\mu} \phi_{\nu} \pm 1\right)\right],
$$

where the sign $( \pm 1)$ is for the moment arbitrary and will be fixed hereafter: the + (resp. - ) sign implies that $\phi^{\mu}$ are the components of a timelike (resp. spacelike) vector. The dynamical variables are the metric $g_{\mu \nu}$ with signature $(-1,+1,+1,+1)$, the scalar field $\phi$ and the extra variable $\lambda$.

\subsubsection{Equations of motion}

The equations of motion are easily computed, and the Euler-Lagrange equations for $\lambda, \phi$ and $g_{\mu \nu}$ are respectively given by

$$
X \pm 1=0, \quad \nabla_{\mu}\left(\lambda \phi^{\mu}\right)=0, \quad G_{\mu \nu}=T_{\mu \nu},
$$

where the stress-energy tensor for the "mimetic" matter field is

$$
T_{\mu \nu}=-2 \lambda \phi_{\mu} \phi_{\nu}+\lambda(X \pm 1) g_{\mu \nu} .
$$

Let us recall that we used the same notations as in the introduction for the gradient $\phi_{\mu} \equiv \nabla_{\mu} \phi$ and the kinetic energy $X \equiv \phi^{\mu} \phi_{\mu}$. The trace of the last equation in (2.2) allows us to express the variable $\lambda$ in terms of the Ricci scalar according to

$$
\lambda= \pm \frac{1}{2} \mathcal{R} .
$$

Substituting this expression for $\lambda$ into the first two equations in (2.2) leads to the following system of equations for the scalar field and the metric

$$
\nabla_{\mu}\left(\mathcal{R} \phi^{\mu}\right)=0, \quad G_{\mu \nu} \pm \mathcal{R} \phi_{\mu} \phi_{\nu}=0 .
$$


Notice that the first equation (for the scalar field) is not independent from Einstein equations. Indeed, the conservation of the Einstein tensor $\nabla^{\mu} G_{\mu \nu}$ together with the mimetic condition necessarily imply that

$$
0=\nabla^{\mu} G_{\mu \nu}=\nabla^{\mu}\left(\mathcal{R} \phi_{\mu} \phi_{\nu}\right)=\nabla^{\mu}\left(\mathcal{R} \phi_{\mu}\right) \phi_{\nu}+\mathcal{R} \phi^{\mu} \phi_{\mu \nu}=\nabla^{\mu}\left(\mathcal{R} \phi_{\mu}\right) \phi_{\nu} \Longrightarrow \nabla_{\mu}\left(\mathcal{R} \phi^{\mu}\right)=0(2.6)
$$

Hence, the equations of motion (2.2) are equivalent to

$$
G_{\mu \nu} \pm \mathcal{R} \phi_{\mu} \phi_{\nu}=0, \quad X \pm 1=0, \quad \lambda= \pm \frac{1}{2} \mathcal{R} .
$$

These equations have been solved for cosmological space-times in [15].

\subsubsection{Generalization: extended mimetic gravity}

The mimetic action (2.1) can be generalized to the form [65]

$$
S\left[g_{\mu \nu}, \phi, \lambda\right] \equiv \int d^{4} x \sqrt{-g}\left[\frac{f(\phi)}{2} \mathcal{R}+L_{\phi}\left(\phi, \chi_{1}, \cdots, \chi_{p}\right)+\lambda(X \pm 1)\right],
$$

where $f$ is an arbitrary function of $\phi, L_{\phi}$ depends on $\phi$ and $\chi_{n}$ which are variables constructed with second derivatives of the scalar field according to

$$
\chi_{n} \equiv \operatorname{Tr}\left([\phi]^{n}\right) \equiv \sum_{\mu_{1}, \cdots, \mu_{n}} \phi_{\mu_{1}}^{\mu_{2}} \phi_{\mu_{2}}^{\mu_{3}} \cdots \phi_{\mu_{n-1}}^{\mu_{n}} \phi_{\mu_{n}}^{\mu_{1}}
$$

Here, we have used the notation $[\phi]$ for the matrix whose coefficients are $[\phi]_{\mu \nu} \equiv \phi_{\mu \nu}$. Indices are lowered and raised by the metric and its inverse. One can show that this extended action defines the most general mimetic gravity like theory $[63,64]$ which propagates at most three degrees of freedom (one scalar in addition to the usual two tensorial modes). Notice that mimetic gravity has recently been generalized in [66] to actions with higher derivatives of the metric (which propagate more than three degrees of freedom out of the unitary gauge). Due to the mimetic condition $X \pm 1=0$, any $X$ dependency in $f$ or $L_{\phi}$ can be removed. More precisely, as it was shown in [64], if one starts with an action (2.8) where $f$ and $L_{\phi}$ depend also on $X$ (and eventually its derivatives $\partial_{\mu} X$ ), the associated equations of motion are equivalent to the equations of motion obtained from the same action where $f$ and $L_{\phi}$ are evaluated to $X=\mp 1$ (and eventually $\partial_{\mu} X=0$ ).

The Euler-Lagrange equations for (2.8) can be easily obtained in full generality. But, for simplicity, we assume that $f$ is a constant (and thus independent of $\phi$ ) which can be fixed to $f=1$. Deriving the action with respect to $\phi$ and $g_{\mu \nu}$ respectively leads to the equations

$$
\frac{\partial L_{\phi}}{\partial \phi}-2 \nabla^{\mu}\left(\lambda \phi_{\mu}\right)+\sum_{n=1}^{p} n \nabla^{\mu \nu}\left([\phi]_{\mu \nu}^{n-1} \frac{\partial L_{\phi}}{\partial \chi_{n}}\right)=0 \quad \text { and } \quad G_{\mu \nu}=T_{\mu \nu}
$$

where $[\phi]^{n}$ is the power $n$ of the matrix $[\phi]$ with the convention $[\phi]_{\mu \nu}^{0} \equiv g_{\mu \nu}$, and now the stress-energy tensor reads

$$
\begin{aligned}
T_{\mu \nu} & =-2 \lambda \phi_{\mu} \phi_{\nu}+\lambda(X \pm 1) g_{\mu \nu}+T_{\mu \nu}^{(\phi)} \\
T_{\mu \nu}^{(\phi)} & \equiv L_{\phi} g_{\mu \nu}+\sum_{n=1}^{p} n\left\{-2 \frac{\partial L_{\phi}}{\partial \chi_{n}}[\phi]_{\mu \nu}^{n}+\nabla^{\alpha}\left[\frac{\partial L_{\phi}}{\partial \chi_{n}}\left([\phi]_{\alpha \mu}^{n-1} \phi_{\nu}+[\phi]_{\alpha \nu}^{n-1} \phi_{\mu}-[\phi]_{\mu \nu}^{n-1} \phi_{\alpha}\right)\right](\boldsymbol{f} .\right.
\end{aligned}
$$


To get rid of $\lambda$ in the Einstein equation, we proceed as in the previous case. First, we take the trace of the second equation in (2.10) to express $\lambda$ in terms of $\phi$ and $g_{\mu \nu}$

$$
\lambda=\mp \frac{1}{2}\left(\mathcal{R}+T^{(\phi)}\right), \quad T^{(\phi)}=4 L_{\phi}-\sum_{n=1}^{p} n\left\{2 \frac{\partial L_{\phi}}{\partial \chi_{n}} \chi_{n}+\nabla^{\alpha}\left[\phi_{\alpha} \frac{\partial L_{\phi}}{\partial \chi_{n}} \chi_{n-1}\right]\right\} .
$$

Then we substitute this expression in the two equations above (2.10). Furthermore, the equation for the scalar field can be obtained from the conservation of the stress-energy tensor, and thus is not independent from Einstein equations.

Hence, as the trace of Einstein equations is trivially satisfied (the trace has been used to determine $\lambda$ ), the equations of motion are equivalent to the mimetic condition and (the traceless part of) Einstein equations only:

$$
X \pm 1=0, \quad G_{\mu \nu}= \pm\left(\mathcal{R}+T^{(\phi)}\right) \phi_{\mu} \phi_{\nu}+T_{\mu \nu}^{(\phi)} .
$$

Solutions to these equations have been studied in the context of cosmology [26] and black holes [10] with a particular choice for $L_{\phi}$ which makes the solutions nonsingular. Here, we focus on black hole solutions and we are going to see how one can choose $L_{\phi}$ to resolve the black hole singularity.

\subsection{Black hole with a limiting curvature}

The non-singular black hole introduced by Chamseddine and Mukhanov in [10] is a "static" spherically symmetric solution of the general mimetic action (2.8) where $L_{\phi}$ is a function of $\chi_{1}$ only defined by

$$
L_{\phi}\left(\chi_{1}\right)=\frac{2}{3} \rho_{m} f(\zeta), \quad \zeta=\frac{\chi_{1}}{\sqrt{\rho_{m}}}, \quad f(\zeta) \equiv 1+\frac{1}{2} \zeta^{2}-\sqrt{1-\zeta^{2}}-\zeta \arcsin \zeta,
$$

where $\rho_{m}$ defines a new energy scale in the theory. This expression of $L_{\phi}$ seems to be an ad hoc choice a priori, but it leads to very appealing nonsingular cosmological and black hole solutions. Notice that, in the cosmological sector, the equation of motion of the scale factor reproduces exactly the effective dynamics of LQC as it was pointed out in $[8,9]$.

Let us now consider a spherically symmetric space-time only, and let us explain physically why (2.15) produces non-singular black hole solutions. For that purpose, we start writing the metric in Schwarzschild coordinates

$$
d s^{2}=-F(R) d T^{2}+\frac{1}{F(R)} d R^{2}+R^{2}\left(d \theta^{2}+\sin ^{2} \theta d \varphi^{2}\right),
$$

where $F$ is a function of $R$ only. In usual general relativity (with no modifications), Einstein equations lead to the Schwarzschild solution where $F(R)=1-2 m / R, m$ being the mass of the black hole. Computing the expansion of outgoing null geodesics in the advanced EddingtonFinkelstein cordinates, non-singular when $F(R)=0$, one obtains $\theta_{+}=F(R) / R$. There exists thus a future trapping horizon at $R=2 \mathrm{~m}$, which is an event horizon due to the staticity of the metric. One can therefore distinguish between the outside $\left(F>0\right.$ or $R>2 m$, thus $\left.\theta_{+}>0\right)$ and the inside of the black hole which is gravitationnally trapped $(F<0$ or $R<2 m$, thus $\left.\theta_{+}<0\right)$. The singularity occurs inside the black hole where the curvature becomes arbitrary large (in the limit $R \rightarrow 0$ ). 
It was shown in the original paper [10] that the action (2.8) with the field Lagrangian (2.15) reproduces correctly the Schwarzschild metric far from the high curvature regions (compared with the scale $\rho_{m}$ ). In particular, spherically symmetric solution (2.16) possesses an event horizon, very similar to the Schwarzschild horizon. Thus one can still define a region inside (or behind) the horizon $(F<0)$ and a region outside the horizon $(F>0)$.

Concerning the scalar field, let us start assuming that it depends on $R$ and $T$ for purposes of generality. We will shortly reduce ourselves to the case of a static scalar field $\phi(R)$. The scalar field satisfies the mimetic condition which reads

$$
-\frac{1}{F(R)}\left(\frac{\partial \phi}{\partial T}\right)^{2}+F(R)\left(\frac{\partial \phi}{\partial R}\right)^{2} \pm 1=0 .
$$

This equation allows to resolve the scalar field $\phi$ in terms of the geometry $F(R)$. A simple class of solutions of this partial differential equation can be obtained from the ansatz

$$
\phi(R, T)=q T+\psi(R),
$$

where $q$ is a constant and $\psi$ satisfies

$$
\left(\frac{d \psi}{d R}\right)^{2}=\frac{q^{2} \mp F}{F^{2}}
$$

Notice that similar ansatz were considered in $[67,68]$ to find black holes and stars solutions in the context of Horndeski (or beyond Horndeski) theories.

It is clear that the equation (2.19) admits a solution only if the condition $q^{2} \mp F \geq 0$ is fulfilled. As a result, in the static case (where $q=0$ ), one cannot find any global spherically symmetric solution for the space-time. Indeed, the condition $\pm F \leq 0$ implies that only the action with $\mathrm{a}+($ resp. - -$)$ sign could lead to a description of the region inside (resp. outside) the black hole. Only a non-static solution for the scalar field $(q \neq 0)$ could enable us to describe a fully static spherically symmetric space-time. However, we will proceed as in [10]: we will restrict ourselves to the region inside the black hole (we expect the limiting curvature hypothesis to affect mainly the regions inside the black hole), we choose a mimetic action with a + sign, and we will argue how this is enough to resolve indeed the singularity. From a phenomenological point of view, we could interpret the action (2.8) with (2.15) as an effective description of general relativity in a region (inside the black hole) where the curvature becomes high (with respect to the scale $\rho_{m}$ ). Such a modification could result from quantum gravity effects for instance [8].

When the scalar field is static, the mimetic condition reduces to a simple differential equation

$$
\left(\frac{d \phi}{d R}\right)^{2}=-\frac{1}{F}
$$

in the region (behind the horizon) where $F \leq 0$ (with appropriate boundary conditions). The form of $L_{\phi}$ (the presence of $\arcsin (x)$ or $\sqrt{1-x^{2}}$ with $x=\chi_{1} / \sqrt{\rho_{m}}$ for instance) imposes that the scalar field $\phi$ must satisfy the condition

$$
\left|\chi_{1}\right| \leq \sqrt{\rho_{m}} \Longrightarrow\left|\frac{d}{d R}\left(R^{2} \sqrt{-F}\right)\right| \leq \sqrt{\rho_{m}} R^{2} .
$$


If one naively substitutes the Schwarzschild solution in this inequality, one gets the condition that

$$
\rho \equiv \frac{m}{R^{3}} \leq \frac{2}{9} \rho_{m},
$$

which can be interpreted by the fact that the density inside the black hole is bounded from above. Hence, one would expect the singularity to be resolved. This has been shown to be indeed the case in [10] from a resolution of the equations of motion. We are going to reproduce this result in the next section from a Hamiltonian point of view.

\section{Hamiltonian description}

In this section, we perform the Hamiltonian analysis of the mimetic action with a limiting curvature. We first introduce the ADM parametrization for the metric and we solve the mimetic condition to integrate out the scalar field $\phi$. Then, we start the Hamiltonian analysis and we find a nice parametrization of the phase space such that the Hamiltonian and vectorial constraints take a rather simple form. Finally, we resolve the Hamilton equations far behind the horizon, and we recover that the solution is indeed non-singular. As we are going to see, the Hamiltonian point of view leads to a simpler analysis of the equations of motion than the Lagrangian point of view, as it was done in [10].

\section{$3.13+1$ decomposition: metric and scalar field}

In this subsection, we start introducing the tools which are necessary to perform the Hamiltonian analysis of the theory restricted to spherically symmetric geometries. Notice that the "radial" and "time" coordinates (in the Schwarzschild parametrization) exchange their roles when one crosses the horizon. In particular, the time coordinate behind the horizon would correspond to the radial coordinate in the Schwarzschild parametrization.

\subsubsection{ADM decomposition for spherical space-time}

We start with the usual ADM decomposition of the (non-static) spherically symmetric metric inside the black hole:

$$
d s^{2}=-N^{2} d t^{2}+\gamma_{r r}\left(d r+N^{r} d t\right)^{2}+\gamma_{\theta \theta} d \Omega^{2}, \quad d \Omega^{2} \equiv d \theta^{2}+\sin ^{2} \theta d \varphi^{2},
$$

where $N(r, t)$ is the lapse function, $N^{r}(r, t)$ is the radial component of the shift vector and

$$
\gamma \equiv \operatorname{diag}\left[\gamma_{r r}(r, t), \gamma_{\theta \theta}(r, t), \gamma_{\theta \theta}(r, t) \sin ^{2} \theta\right]
$$

are the non-vanishing components of the (spherically symmetric) induced metric on the three dimensional space-like hypersurface. In the following, we will use the standard notations $\gamma^{r r} \equiv \gamma_{r r}^{-1}$ and $\gamma^{\theta \theta} \equiv \gamma_{\theta \theta}^{-1}$ for the components of the inverse metric $\gamma^{-1}$.

\subsubsection{Resolution of the mimetic condition to integrate out the scalar field}

Concerning the scalar field, we assume that it depends on time $t$ only (which corresponds to a "static" solution from the point of view of an observer outside the horizon). The mimetic condition $X+1=0$ implies that the lapse function necessarily depends on $t$ according to

$$
\dot{\phi}(t)^{2}=N(t)^{2} .
$$


Without loss of generality, we take the solution $\dot{\phi}=+N$ that we substitute in the action in order to integrate out the scalar field $\phi$. To do so, we also need to compute $\chi_{1}=\square \phi$ in terms of the metric variables.

Thus, we start by computing second derivatives of the scalar field $\phi_{\mu \nu}$, and an easy calculation shows that the only non-vansihing components of $\phi_{\mu \nu}$ are

$$
\phi_{t t}=-\left(N^{r}\right)^{2} K_{r r}, \quad \phi_{r t}=-N^{r} K_{r r}, \quad \phi_{i i}=-K_{i i},
$$

where $i \in\{r, \theta, \varphi\}$ labels spatial coordinates, and $K_{i j}$ are the components of the extrinsic curvature

$$
K_{i j} \equiv \frac{1}{2 N}\left(\dot{\gamma}_{i j}-D_{i} N_{j}-D_{j} N_{i}\right)
$$

with $D_{i}$ being the covariant derivative compatible with the spatial metric $\gamma_{i j}$ (3.2). To go further, one has to compute explicitly the components of the extrinsic curvature. Only the diagonal components are non-trivial with

$$
K_{r r}=\frac{1}{2 N}\left(\dot{\gamma}_{r r}-\gamma_{r r}^{\prime} N^{r}-2 \gamma_{r r}\left(N^{r}\right)^{\prime}\right), \quad K_{\theta \theta}=\frac{K_{\varphi \varphi}}{\sin ^{2} \theta}=\frac{1}{2 N}\left(\dot{\gamma_{\theta}}-\gamma_{\theta \theta}^{\prime} N^{r}\right) .
$$

As usual, dot and prime denote respectively time and radial derivatives. We have now all the ingredients to start the Hamiltonian analysis of the theory.

\subsection{Hamiltonian analysis}

Using the Gauss-Codazzi relation, the action (2.1) with $f(\phi)=1$ and $L_{\phi}$ given by (2.15) reduces to the form

$$
S=\int d t d^{3} x N \sqrt{\gamma}\left[\frac{1}{2}\left(K_{i j} K^{i j}-K^{2}+R\right)+L_{\phi}(K)\right],
$$

where we have substituted the solution of the mimetic constraint for the scalar field (3.4), and $R$ is the three-curvature whose expression in terms of the metric components is

$$
R=\frac{2}{\gamma_{\theta \theta}}\left[1-\left(\frac{\gamma_{\theta \theta}^{\prime}}{\sqrt{\gamma_{\theta \theta} \gamma_{r r}}}\right)^{2}+\frac{2}{\gamma_{r r}}\left(\frac{\gamma_{\theta \theta}^{\prime}}{\sqrt{\gamma_{\theta \theta} \gamma_{r r}}}\right)^{\prime}\right]
$$

To simplify the Hamiltonian analysis, it is more convenient to introduce a new parametrization of the metric in terms of the variables $\alpha$ and $\gamma$ defined by

$$
\left\{\gamma \equiv \gamma_{r r} \gamma_{\theta \theta}^{2} \sin ^{2}(\theta), \quad \alpha \equiv \frac{\gamma_{r r}}{\gamma_{\theta \theta}}\right\} \Longleftrightarrow\left\{\gamma_{r r}=\left(\gamma \alpha^{2}\right)^{1 / 3}, \quad \gamma_{\theta \theta}=\left(\frac{\gamma}{\alpha}\right)^{1 / 3}\right\} .
$$

With these new variables, the action simplifies and becomes

$$
S=\int d t d^{3} x \sqrt{\gamma}\left[\frac{1}{12 N}\left(A^{2}-B^{2}\right)+N\left(\frac{R}{2}+L_{\phi}\left(\frac{B}{2 N}\right)\right)\right]
$$

where $A$ and $B$ depend respectively on the variables $\left(\alpha, N^{r}\right)$ and $\left(\gamma, N^{r}\right)$ according to

$$
A \equiv \frac{\dot{\alpha}}{\alpha}-\frac{\alpha^{\prime}}{\alpha} N^{r}-2\left(N^{r}\right)^{\prime}, \quad B \equiv \frac{\dot{\gamma}}{\gamma}-\frac{\gamma^{\prime}}{\gamma} N^{r}-2\left(N^{r}\right)^{\prime} .
$$


Notice that $R$ can be expressed explicitly in terms of $\alpha$ and $\gamma$, what we will not do here because its explicit form is not needed for our purposes.

It is clear from this expression of the Lagrangian (3.10) that $N$ and $N^{r}$ are Lagrange multipliers, and there are only two pairs of conjugate variables defined by the Poisson brackets

$$
\left\{\alpha(u), \pi_{\alpha}(v)\right\}=\delta(u-v)=\left\{\gamma(u), \pi_{\gamma}(v)\right\} .
$$

Momenta are easily computed and are given in terms of the velocities by

$$
\pi_{\alpha}=\frac{1}{3} \frac{\sqrt{\gamma}}{N \alpha} A, \quad \pi_{\gamma}=-\frac{1}{3} \sqrt{\frac{\rho_{m}}{\gamma}} \arcsin \left(\frac{B}{2 N \sqrt{\rho_{m}}}\right) .
$$

Inverting these relations to obtain velocities in terms of momenta is immediate

$$
\begin{aligned}
& \frac{\dot{\alpha}}{\alpha}=3 \frac{N \alpha}{\sqrt{\gamma}} \pi_{\alpha}+\frac{\alpha^{\prime}}{\alpha} N^{r}+2\left(N^{r}\right)^{\prime}, \\
& \frac{\dot{\gamma}}{\gamma}=-2 N \sqrt{\rho_{m}} \sin \left(3 \sqrt{\frac{\rho_{m}}{\gamma}} \pi_{\gamma}\right)+\frac{\gamma^{\prime}}{\gamma} N^{r}+2\left(N^{r}\right)^{\prime} .
\end{aligned}
$$

From these expressions, one easily deduces the expression of the Hamiltonian

$$
H=\int d r\left(N \mathcal{H}+N^{r} D_{r}\right)
$$

with the Hamiltonian and vectorial constraints respectively given by

$$
\begin{aligned}
\mathcal{H} & \equiv \frac{3}{\sqrt{\gamma}} \alpha^{2} \pi_{\alpha}^{2}-\sqrt{\gamma}\left[\frac{4}{3} \rho_{m} \sin ^{2}\left(\frac{3}{2} \sqrt{\frac{\gamma}{\rho_{m}}} \pi_{\gamma}\right)+\frac{1}{2} R\right], \\
D_{r} & \equiv-\left(\gamma^{\prime} \pi_{\gamma}+2 \gamma \pi_{\gamma}^{\prime}+\alpha^{\prime} \pi_{\alpha}+2 \alpha \pi_{\alpha}^{\prime}\right) .
\end{aligned}
$$

Hence, time derivative of any functionnal $\mathcal{O}$ of the phase space variables is computed from the Poisson bracket

$$
\dot{\mathcal{O}}(t, r)=\{\mathcal{O} ; H\}
$$

from which one easily deduces the equations of motion. To these equations, one adds the two constraints $\mathcal{H} \approx 0$ and $D_{r} \approx 0$ to integrate completely the system. Notice that $\approx$ denotes the weak equality in the phase space.

Notice that the Hamiltonian constraint can be written as

$$
\rho \equiv \frac{9 \alpha^{2}}{4 \gamma} \pi_{\alpha}^{2}-\frac{3}{8} R \approx \rho_{m} \sin ^{2}\left(\frac{3}{2} \sqrt{\frac{\gamma}{\rho_{m}}} \pi_{\gamma}\right),
$$

where $\rho$ will be interpreted as the energy density of the mimetic scalar field, as we are going to argue later. The density $\rho$ is necessarily bounded by $\rho_{m}$.

\subsection{Spherically "static" solutions deeply inside the black hole}

To go further, we restrict ourselves to spherically static solutions which correspond, in the black hole interior, to having a time dependency only. 


\subsubsection{Equations of motion}

In that case, the vectorial constraint $D_{r}=0$ is strongly satisfied and the expression of the 3 -dimensional Ricci scalar reduces to $R=2(\alpha / \gamma)^{1 / 3}$. Hence, after some simple calculations, one shows that the equations of motion for the phase space variables are

$$
\begin{aligned}
& \dot{\alpha}=\frac{6 \alpha^{2} \pi_{\alpha}}{\sqrt{\gamma}}, \quad \dot{\pi}_{\alpha}=-\frac{6 \alpha \pi_{\alpha}^{2}}{\sqrt{\gamma}}+\frac{1}{3} \frac{\gamma^{1 / 6}}{\alpha^{2 / 3}}, \quad \dot{\gamma}=-2 \gamma \sqrt{\rho_{m}} \sin \left(3 \sqrt{\frac{\gamma}{\rho_{m}}} \pi_{\gamma}\right), \\
& \dot{\pi}_{\gamma}=\frac{3}{2} \frac{\alpha^{2} \pi_{\alpha}^{2}}{\gamma^{2 / 3}}+\frac{\rho_{m}}{3 \sqrt{\gamma}} \sin ^{2}\left(\frac{3}{2} \sqrt{\frac{\gamma}{\rho_{m}}} \pi_{\gamma}\right)+\frac{\rho_{m}}{2} \pi_{\gamma} \sin \left(3 \sqrt{\frac{\gamma}{\rho_{m}}} \pi_{\gamma}\right)-\frac{\alpha^{1 / 3}}{6 \gamma^{5 / 3}},
\end{aligned}
$$

where we have fixed the lapse to the value $N=1$ for simplicity (which corresponds to a redefinition of the time variables). These equations are highly non-linear and very cumbersome to solve. Even though we do not expect to find explicit solutions, we are going to present some interesting properties they satisfy.

First of all, the equation for $\gamma$ (3.21) together with the Hamiltonian constraint (3.20) leads to the so-called master equation in [10] given by

$$
\left(\frac{\dot{\gamma}}{4 \gamma}\right)^{2}=\rho\left(1-\frac{\rho}{\rho_{m}}\right), \quad \text { with } \rho=\frac{1}{4}\left[\frac{1}{4}\left(\frac{\dot{\alpha}}{\alpha}\right)^{2}-3\left(\frac{\alpha}{\gamma}\right)^{1 / 3}\right] \text {. }
$$

In [10], the same equation has been obtained from the Lagrangian point of view in terms of the variable $\epsilon \equiv(4 / 3) \rho$ and the constant $\epsilon_{m} \equiv(4 / 3) \rho_{m}$. We recover an equation very similar to the one satisfied by the scale factor in the framework of effective LQC [8], which leads to a non-singular scenario for early universe cosmology. Here, we have a similar dynamics for $\gamma$ which is also bounded from below: this leads to a non-singular black hole solution [10] as it can be easily seen from the expression of the Kretschmann tensor $\mathcal{K}=4(\alpha / \gamma)^{1 / 3}$ : it is bounded when $\gamma$ does not vanish, provided that $\alpha$ does not tend to infinity neither.

\subsubsection{Deep inside the black hole: resolution of the singularity}

To understand better the dynamics of the variables $\alpha$ and $\gamma$, let us consider the regime where the 3-dimensional scalar curvature $R$ becomes negligible in the expression of the energy density (3.20). This hypothesis implies the condition

$$
\frac{\alpha^{2} \pi_{\alpha}^{2}}{\gamma} \gg R \Longleftrightarrow\left(\frac{\dot{\alpha}}{\alpha}\right)^{2} \gg\left(\frac{\alpha}{\gamma}\right)^{1 / 3}
$$

It has been shown in [10] that this condition is satisfied deeply inside the black hole (in the region where the quantum effects are supposed to be important). In this situation, the equations for $\alpha$ and $\pi_{\alpha}$ become

$$
\dot{\alpha}=\frac{6 \alpha^{2} \pi_{\alpha}}{\sqrt{\gamma}}, \quad \dot{\pi}_{\alpha}=-\frac{6 \alpha \pi_{\alpha}^{2}}{\sqrt{\gamma}},
$$

which can be integrated exactly. First, $\alpha$ can be expressed in terms of $\gamma$ using the relations:

$$
\sqrt{\gamma} \frac{\dot{\alpha}}{\alpha}=C \quad \Longleftrightarrow \quad \alpha(t)=\alpha_{0} \exp \left(C \int_{0}^{t} \frac{d u}{\sqrt{\gamma(u)}}\right)
$$


where $C$ and $\alpha_{0}$ are integration constants. Then, the equation for $\gamma(3.23)$ simplifies and becomes

$$
\dot{\gamma}^{2}=C^{2}\left(\gamma-\gamma_{m}\right), \quad \gamma_{m} \equiv \frac{C^{2}}{16 \rho_{m}},
$$

from which we deduce immediately that $\gamma$ is bounded from below by $\gamma_{m}$. Furthermore, one easily integrates this equation and obtains an exact expression for $\gamma$ (in the regime where $R$ is negligible in the expression of $\mathcal{H}_{0}$ ) given by

$$
\gamma(t)=\gamma_{m}\left(1+4 \rho_{m} t^{2}\right),
$$

assuming that $\gamma(0)=\gamma_{m}$. This expression coincides completely with Eq.(64) of [10] when the constant $C$ has been fixed to $C=3 r_{g}$ ( $r_{g}$ being the Schwarzchild radius). Finally, substituting this expression in (3.26), one obtains

$$
\alpha(t)=a \exp \left[2 \sinh ^{-1}\left(2 \sqrt{\rho_{m}} t\right)\right]
$$

where $a$ is a new constant. Hence, we recover exactly the solution found in [10] where $a$ has been fixed to

$$
a=\frac{64}{9} \rho_{m}
$$

As a conclusion, the singularity is clearly avoided and replaced by a bounce very similar to the LQC bounce.

Let us recall that the analysis of this subsection is valid only when the condition (4.50) is fulfilled. Using the relation (3.26), the validity condition can be more explicitly given by

$$
C^{6} \gg \alpha \gamma^{2} \Longleftrightarrow 18^{2} r_{g}^{2} \rho_{m} \gg\left(1+4 \rho_{m} t^{2}\right)^{2} \exp \left[2 \sinh ^{-1}\left(2 \sqrt{\rho_{m}} t\right)\right] .
$$

Thus, $\rho_{m} t^{2} \ll 1$ is sufficient for the condition (3.31) to be satisfied provided that

$$
Q \equiv r_{g}^{2} \rho_{m} \gg 1
$$

which is obviously the case deep inside the black hole. However, this is not necessary. Indeed, in the regime where $\rho_{m} t^{2} \gg 1$, the previous condition gives

$$
\rho_{m} t^{3} \ll r_{g},
$$

which is less restrictive than $\rho_{m} t^{2} \ll 1$. Hence, the solution (deep inside the black hole) given by (3.28) and (3.29) is valid when $t$ is sufficiently small according to (3.33).

\subsubsection{Comparing with the Schwarzschild solution}

To conclude this analysis of the geometry deep inside the black hole, let us make a comparison with the usual Schwarzschild solution. When expressed in terms of the parameters $\alpha$ and $\gamma$, the Schwarzschild black hole is defined by (see [10] for instance)

$$
\alpha_{s}=\frac{1-\tau^{2}}{r_{g}^{2} \tau^{6}}, \quad \gamma_{s}=r_{g}^{4}\left(1-\tau^{2}\right) \tau^{6} \quad \text { with } \quad \frac{t}{r_{g}} \equiv \arcsin \tau-\tau \sqrt{1-\tau^{2}}
$$




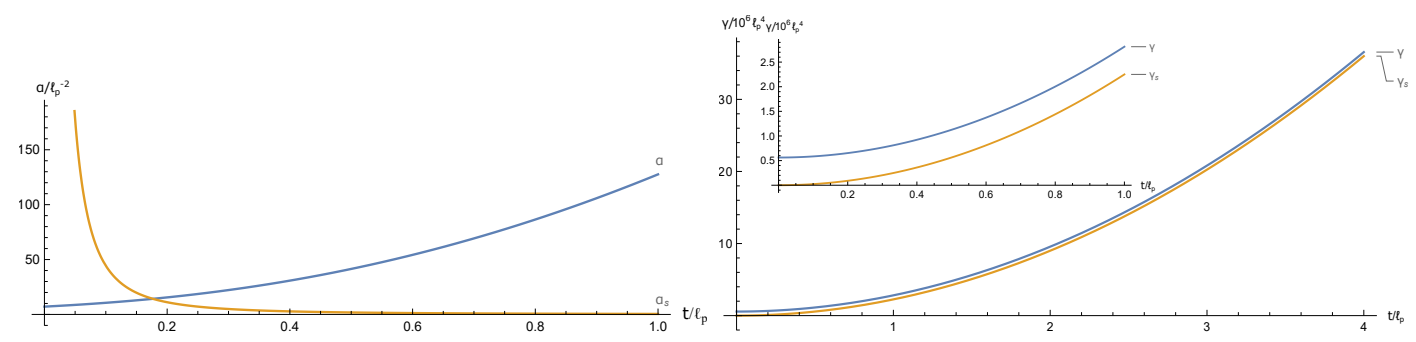

Figure 1. These two graphs show the solutions $\alpha(t)$ and $\gamma(t)$ compared to the Schwarzschild solution $\alpha_{s}(t)$ and $\gamma_{s}(t)$ in the deep quantum regime (3.33). In particular, we see that the limiting curvature effect makes the functions $\alpha(t)$ and $1 / \gamma(t)$ no more divergent when $t \rightarrow 0$, which regularizes the singularity. In these plots, we work in the Planck unit with $r_{g}=1000 \ell_{p}$ and $\rho_{m}=\ell_{p}^{-2}$, where $\ell_{p}$ is the Planck length. In that case, the deep quantum regime condition $(3.33)$ becomes $t \ll 10 \ell_{p}$.

In the regime (3.33), we necessarily have $t / r_{g} \ll Q^{-1 / 3} \ll 1$, hence $\tau \ll 1$ and then

$$
\tau^{3} \approx \frac{3 t}{2 r_{g}}
$$

Thus, the Schwarzschild solution, to compare the regularized solution with, reduces to

$$
\alpha_{s}(t) \approx \frac{4}{9 t^{2}}, \quad \gamma_{s}(t) \approx \frac{9}{4} r_{g}^{2} t^{2},
$$

in the region where the curvature is high. We see that both $\alpha$ and $\gamma^{-1}$ are regularized compared to the classical Schwarzschild solution as shown in Fig. (1): this is the effect of the limiting curvature. However, close to the horizon (which corresponds to $\tau \approx 1$ ), one recovers the Schwarzschild solution because the limiting curvature effect becomes negligible.

\section{On the relation with effective polymer black holes}

In $[8,9]$, it was shown that extended mimetic gravity with $(2.15)$ reproduces the dynamics of effective LQC when restricted to homogenous and isotropic cosmology. A natural question is whether this relation extends itself to the spherically symmetric sector. We explore this question in this section where we compare the Hamiltonian of the non-singular black hole with limiting curvature with the standard polymer model of vacuum spherically symmetric gravity in terms of Ashtekar-Barbero variables studied in [11]. While the relation between the Chamseddine-Mukhanov mimetic theory with limiting curvature and LQC is shown not to hold beyond homogenous geometries, one can propose new mimetic theories to describe a generalized regularization for polymer models which provides us with an undeformed notion of covariance as well as a natural inbuilt $\bar{\mu}$-scheme.

\subsection{Spherically symmetric polymer models: a short review}

In this section, we first give a brief review on the formulation of the classical spherically symmetric Ashtekar-Barbero phase space and then we describe the so-called holonomy corrected phase space. As explained in the introduction, this leads to an effective polymer model which has been the starting point for a quantization of spherically symmetric geometries using the LQG techniques [11]. This phase space, which describes vacuum spherically symmetric gravity, has only global degrees of freedom. The quantum effective corrections are introduced 
via holonomy corrections which ensure the existence of an anomaly-free (albeit deformed) Dirac's hypersurface deformation algebra $[1,6]$. In that sense, the resulting polymer phase space describes a vacuum geometry with somehow a deformed notion of covariance.

\subsubsection{The classical vacuum spherically symmetric Ashtekar-Barbero phase space}

Starting from the full Ashtekar-Barbero phase space, one can impose spherical symmetry to reduce it. This symmetry reduction has been presented in [69] and we shall not reproduce the different steps in details but only sketch the main lines.

The spherically symmetric Ashtekar-Barbero phase space is parametrized by two pairs of canonical conjugate variables (after having gauge fixed the Gauss constraint) given by

$$
\left\{K_{x}(x), E^{x}(y)\right\}=\frac{2 \kappa}{\beta} \delta(x-y), \quad\left\{K_{\phi}(x), E^{\phi}(y)\right\}=\frac{\kappa}{\beta} \delta(x-y),
$$

where $1 / \beta$ is the Barbero-Immirzi parameter and $\kappa=8 \pi G$ with $G$ the newton constant. Note the $x$ and $y$ are radial coordinates and $\delta(x-y)$ denotes the usual one-dimensional delta distribution.

The first pair corresponds to the inhomogenous component of the connection with its associated electric field, while the second one is the angular contribution built from the angular components of the connection. As usual in the LQG litterature, $x$ denotes the radial direction as shown in the expression of the induced metric

$$
d s^{2} \equiv \gamma_{r r} d r^{2}+\gamma_{\theta \theta} d \Omega^{2} \equiv \frac{\left(E^{\phi}\right)^{2}}{\left|E^{x}\right|} d x^{2}+\left|E^{x}\right| d \Omega^{2}
$$

The first class constraints generating the gauge symmetries (i.e. spatial diffeomorphisms and time reparametrization) are

$$
\begin{aligned}
& D\left[N^{x}\right]=\frac{1}{2 \kappa} \int_{\Sigma} d x N^{x}\left[2 E^{\phi} K_{\phi}^{\prime}-K_{x}\left(E^{x}\right)^{\prime}\right] \\
& \mathcal{H}[N]=-\frac{1}{2 \kappa} \int_{\Sigma} d x N\left|E^{x}\right|^{-1 / 2}\left[E^{\phi} K_{\phi}^{2}+2 K_{\phi} K_{x} E^{x}+\left(1-\Gamma_{\phi}^{2}\right) E^{\phi}+2 \Gamma_{\phi}^{\prime} E^{x}\right]
\end{aligned}
$$

where the spin-connection component which shows up in the expression of $\mathcal{H}[\mathrm{N}]$ is given by $\Gamma_{\phi} \equiv-\left(E^{x}\right)^{\prime} /\left(2 E^{\phi}\right)$. The two constraints satisfy obviously the hypersurface deformation algebra of general relativity (after reduction to the spherically symmetric geometries)

$$
\begin{aligned}
& \left\{D\left[N^{x}\right], D\left[M^{x}\right]\right\}=D\left[\mathcal{L}_{N} M\right] \\
& \left\{D\left[N^{x}\right], \mathcal{H}[M]\right\}=-\mathcal{H}\left[\mathcal{L}_{N} M\right] \\
& \{\mathcal{H}[N], \mathcal{H}[M]\}=D\left[\gamma^{x x}\left(N \partial_{x} M-M \partial_{x} N\right)\right]
\end{aligned}
$$

where we used the same notations as in the equations (1.4) and (1.5) in the introduction.

\subsubsection{The holonomy corrected polymer phase space}

Following the strategy used to study polymer models at the effective level, one introduces holonomy corrections (called loop corrections) to work with holonomies instead of the connection itself. These effective corrections originate from a general regularization procedure 
borrowed from Loop Quantum Gravity ${ }^{2}$. However, several new difficulties show up when considering an inhomogenous background such as vacuum spherically symmetric gravity compared to homogenous cosmological backgrounds. In particular, one has to ensure that the loop regularization does not generate anomalies in the first class constraints algebra.

The usual regularization consists in making the replacement

$$
K_{\phi} \longrightarrow f\left(K_{\phi}\right)
$$

directly in the expression of the Hamiltonian constraint, where the function $f$ encodes quantum gravity effects at the effective level. Such a procedure is obviously not unique and suffers from ambiguities. Furthermore, there could be as many different functions $f$ as many $K_{\phi}$ that appear in the Hamiltonian constraint. Yet, a standard choice (in the LQG litterature) is

$$
f\left(K_{\phi}\right)=\frac{\sin \left(\rho K_{\phi}\right)}{\rho},
$$

where $\rho$ is a new fundamental (quantum gravity) scale, taken to be the minimal area gap for LQG, such that the limit $\rho \rightarrow 0$ reproduces the classical phase space. Notice that this choice is motivated by the polymerization obtained in LQC, from the computation of the non local curvature operator using $\mathrm{SU}(2)$ holonomies (one can refer to [29] for more details).

Moreover, the above effective corrections depend only on the extrinsic curvature while the parameter $\rho$, i.e. the so called polymer scale, is a constant. This type of correction is known in the terminology of LQC to correspond to the $\mu_{0}$-scheme. In the early stages of LQC, this scheme was used to develop the theory. Yet, it was soon realized that such corrections spoil the semi-classical consistency of the model. Indeed, within this $\mu_{0^{-}}$-scheme, the bounce of the universe as described by LQC could take place at any arbitrary energy density. The way out of this important difficulty was to introduce an improved dynamics based on a new type of effective holonomy corrections, the so called $\bar{\mu}$-scheme. In this new regularization, the polymer scale $\rho$ depends explicitly on the metric. Contrary to the $\mu_{0}$-scheme, where the semi-classical limit had to be taken by hand, i.e. $\rho \rightarrow 0$, the $\bar{\mu}$-scheme allows the polymer scale to run with the metric, and thus $\rho$ naturally vanishes in the large volume limit, as expected from a consistent regularization.

A first attempt to introduce a $\bar{\mu}$-scheme in spherically symmetric loop models has been presented in [7]. In that case, $f$ is now a function $f\left(K_{\phi}, E^{x}\right)$ of $K_{\phi}$ and $E^{x}$. It was shown that the resulting quantum corrections are not periodic anymore if one requires anomaly-freeness of the first class constraints algebra, challenging the possibility to interpret such corrections as resulting fundamentally from $\mathrm{SU}(2)$ holonomy corrections.

With this standard regularization (4.8), the holonomy corrected first class constraints take the form

$$
\begin{aligned}
& D\left[N^{x}\right]=\frac{1}{2 \kappa} \int_{\Sigma} d x N^{x}\left[2 E^{\phi} K_{\phi}^{\prime}-K_{x}\left(E^{x}\right)^{\prime}\right], \\
& \mathcal{H}[N]=-\frac{1}{2 \kappa} \int_{\Sigma} d x N\left|E^{x}\right|^{-1 / 2}\left[E^{\phi} f_{1}\left(K_{\phi}\right)+2 f_{2}\left(K_{\phi}\right) K_{x} E^{x}+\left(1-\Gamma_{\phi}^{2}\right) E^{\phi}+2 \Gamma_{\phi}^{\prime} E^{x}\right],
\end{aligned}
$$

\footnotetext{
${ }^{2}$ Note that the full regularization implies additional corrections: the triad corrections associated to the regularization of the inverse volume term. Yet, most of the investigations only implement one type of corrections while ignoring the second type for practical reasons. Even more, among the holonomy corrections, only the point wise quantum corrections are taken into account. Beyond the technical aspect, the motivation for focusing on the holonomy corrections only comes from the observation that in the cosmological sector, such corrections are enough to obtain a singularity resolution mechanism.
} 
where the two functions $f_{1}$ and $f_{2}$ regularize the two $K_{\phi}$ arguments which appear in the expression of the classical Hamiltonian constraint. The corresponding Poisson algebra is

$$
\begin{aligned}
& \left\{D\left[N^{x}\right], D\left[M^{x}\right]\right\}=D\left[\mathcal{L}_{N} M\right], \\
& \left\{D\left[N^{x}\right], \mathcal{H}[M]\right\}=-\mathcal{H}\left[\mathcal{L}_{N} M\right], \\
& \{\mathcal{H}[N], \mathcal{H}[M]\}=D\left[\beta\left(K_{\phi}\right) \gamma^{x x}\left(N \partial_{x} M-M \partial_{x} N\right)\right],
\end{aligned}
$$

provided that the two corrections functions satisfy the differential equation

$$
f_{2}=\frac{1}{2} \frac{d f_{1}}{d K_{\phi}}
$$

This implies in turn that the deformation of the Dirac's algebra is given by

$$
\beta\left(K_{\phi}\right)=\frac{d f_{2}}{d K_{\phi}} .
$$

Interestingly, at the bounce, the sign of the function $\beta\left(K_{\phi}\right)$ changes (when one takes the usual sin polymerization function (4.9)) and the constraints algebra becomes effectively euclidean. This observation has suggested a possible signature change of the space-time in the very quantum region. This point is still debated and has received more attention in the context of the deformed algebra approach to the cosmological perturbations in LQC [70, 71]. See [72-74] for a general discussion on the conceptual and technical consequences of this deformation in early cosmology, and [75] in the context of black hole. Finally, note that the above deformation disappears when working with the self-dual Ashtekar variables. Indeed, thanks to the the self-dual formulation, one can introduce point wise holonomy corrections with a $\bar{\mu}$-scheme without affecting the Dirac's algebra which keeps its classical form. This was shown both for spherically symmetric gravity coupled to a scalar field and for the unpolarized Gowdy model, which both exhibit local degrees of freedom $[38,39]^{3}$.

In the case of a real Ashtekar-Barbero spherically symmetric polymer phase space, the usual choice for an effective description of spherically symmetric geometries in LQG corresponds to taking

$$
f_{1}\left(K_{\phi}\right)=\frac{\sin ^{2}\left(\rho K_{\phi}\right)}{\rho^{2}}, \quad f_{2}\left(K_{\phi}\right)=\frac{\sin \left(2 \rho K_{\phi}\right)}{2 \rho},
$$

which obviously satisfies (4.15).

\subsubsection{Comparing mimetic and polymer black holes}

Now, we compare the Hamiltonian structures of LQG and mimetic gravity with a limiting curvature. Following the previous section, we can write the effective constraints of spherically symmetric LQG as follows:

$$
\begin{aligned}
& \mathcal{H}=\left|E^{x}\right|^{-1 / 2}\left[E^{\phi} \frac{\sin ^{2}\left(\rho K_{\phi}\right)}{\rho^{2}}+2 \frac{\sin \left(2 \rho K_{\phi}\right)}{2 \rho} K_{x} E^{x}+\left(1-\Gamma_{\phi}^{2}\right) E^{\phi}+2 \Gamma_{\phi}^{\prime} E^{x}\right], \\
& D_{x}=2 E^{\phi} K_{\phi}^{\prime}-K_{x}\left(E^{x}\right)^{\prime} .
\end{aligned}
$$

To make a comparison with mimetic gravity in the spherical sector, we need to reformulate the mimetic phase space (3.12) and the mimetic constraints (3.17) in terms of the LQG

\footnotetext{
${ }^{3}$ See also [76] for similar conclusion in the inhomogeneous cosmological context.
} 
variables. Let us start with the phase space variables. First of all, the coordinates $\alpha$ and $\gamma$ are easily related to the components $E^{\phi}$ and $E^{x}$ of the electric field as follows:

$$
\alpha=\gamma_{r r} \gamma_{\theta \theta}^{-1}=\left(E^{\phi}\right)^{2}\left(E^{x}\right)^{-2}, \quad \gamma=\gamma_{r r} \gamma_{\theta \theta}^{2}=\left(E^{\phi}\right)^{2} E^{x} .
$$

To obtain the relation between the momenta $\left(\pi_{\alpha}, \pi_{\gamma}\right)$ and the components $\left(K_{\phi}, K_{x}\right)$ of the extrinsic curvature, we transform the symplectic potential $\Theta$ of the LQG phase space

$$
\Theta \equiv \beta\left[K_{\phi} \delta E^{\phi}+\frac{1}{2} K_{x} \delta E^{x}\right]
$$

as follows

$$
\begin{aligned}
\Theta & =\beta\left[K_{\phi} \delta\left(\alpha^{\frac{1}{6}} \gamma^{\frac{1}{3}}\right)+\frac{1}{2} K_{x} \delta\left(\alpha^{-\frac{1}{3}} \gamma^{\frac{1}{3}}\right)\right], \\
& =\beta\left[\frac{K_{\phi}}{3 E^{\phi} E^{x}}+\frac{K_{x}}{6\left(E^{\phi}\right)^{2}}\right] \delta \gamma+\beta\left[\frac{K_{\phi}\left(E^{x}\right)^{2}}{6 E^{\phi}}-\frac{K_{x}\left(E^{x}\right)^{3}}{6\left(E^{\phi}\right)^{2}}\right] \delta \alpha .
\end{aligned}
$$

Due to the equality $\Theta=\pi_{\gamma} \delta \gamma+\pi_{\alpha} \delta \alpha$, we deduce immediately the useful relations

$$
\pi_{\alpha}=\beta\left[\frac{K_{\phi}\left(E^{x}\right)^{2}}{6 E^{\phi}}-\frac{K_{x}\left(E^{x}\right)^{3}}{6\left(E^{\phi}\right)^{2}}\right], \quad \pi_{\gamma}=\beta\left[\frac{K_{\phi}}{3 E^{\phi} E^{x}}+\frac{K_{x}}{6\left(E^{\phi}\right)^{2}}\right] .
$$

Substituting (4.20) and (4.24) in (3.17), one immediately obtains the expressions of the mimetic Hamiltonian constraint in terms of the LQG variables

$$
\mathcal{H}=\frac{\beta^{2}}{12} \frac{\left(K_{\phi} E^{\phi}-K_{x} E^{x}\right)^{2}}{E^{\phi} \sqrt{E^{x}}}-\frac{4 \rho_{m}}{3} E^{\phi} \sqrt{E^{x}} \sin ^{2}\left(\frac{\beta}{6} \frac{2 K_{\phi} E^{\phi}+K_{x} E^{x}}{\sqrt{\rho_{m}} E^{\phi} \sqrt{E^{x}}}\right)-\frac{1}{2} E^{\phi} \sqrt{E^{x}} R .
$$

Hence, the Hamiltonian constraints of mimetic gravity and LQG do not not coincide. The identification we have noticed for homogeneous and isotropic backgrounds does not extend to spherically symmetric geometries. While only the variable $K_{\phi}$ is polymerized in the polymer Hamiltonian (using a constant scale $\rho$ ), it is a rather complicated combination of the different fields that enters in the sine function in mimetic gravity, namely

$$
\mathcal{A}=\tilde{\rho}\left(K_{\phi}+\frac{E^{x}}{E^{\phi}} K_{x}\right) \quad \text { with } \quad \tilde{\rho}=\frac{\beta}{3 \sqrt{\rho_{m} E^{x}}} .
$$

Notice that in this last quantity, $\tilde{\rho}$ depends on the phase space field $E^{x}$, and provides a natural $\bar{\mu}$-scheme.

The result obtained in this section is not surprising. Indeed, since the effective polymer model has a deformed notion of covariance compared with General Relativity, one does not expect to find a covariant effective action which would reproduce its Hamiltonian formulation. However, one can use mimetic gravity with a limiting curvature as a guide to build undeformed covariant notion of point-wise holonomy like corrections. Therefore, while we do not expect to reproduce any existing inhomogeneous polymer models from these scalar-tensor theories (albeit in the cosmological sector where the issue of covariance disappears), we want to build new extended mimetic Lagrangians which reproduce "as closely as possible" the Hamiltonian of the current spherically symmetric polymer models. The difference between the mimetic and polymer Hamiltonian formulations will then provide an interesting guide to understand the absence of covariance in inhomogeneous polymer black holes. The next section is devoted to this task. 


\subsection{Polymer Hamiltonians from extended mimetic gravity}

In this section, we present a method such that, given effective corrections introduced at the hamiltonian level in the spherically symmetric phase space of vacuum gravity, one can reconstruct the associated extended mimetic covariant Lagrangian. The theory remains fully covariant despite the effective hamiltonian corrections and thus extended mimetic gravity provides us with a guide to build a covariant regularization of the classical phase space of spherically symmetric gravity.

\subsubsection{General construction}

Our starting point is again the most general form for the extended mimetic action (2.8)

$$
S\left[g_{\mu \nu}, \phi, \lambda\right] \equiv \int d^{4} x \sqrt{-g}\left[\frac{f(\phi)}{2} \mathcal{R}+L_{\phi}\left(\phi, \chi_{1}, \cdots, \chi_{p}\right)+\lambda(X \pm 1)\right],
$$

where $f$ is an arbitrary function of $\phi$, and $L_{\phi}$ depends on $\phi$ and $\chi_{n}$ defined in (2.9). For simplicity, we choose $f=1$ and we assume that $L_{\phi}$ does not depend on $\phi$.

Thanks to the mimetic constraint, in the "static" case where the scalar field depends on time $t$ only, one can relate directly the second order derivative of the scalar field $\phi_{\mu \nu}$ to the extrinsic curvature of the 3-hypersurface $\Sigma$ of the ADM decomposition. Using (3.4), one can rewrite the variable $\chi_{n}$ as

$$
\chi_{n}=(-1)^{n} \sum_{i_{1}, \ldots, i_{n}} K_{i_{1}}^{i_{2}} K_{i_{2}}^{i_{3}} \ldots K_{i_{n-1}}^{i_{n}} K_{i_{n}}^{i_{1}} .
$$

In the spherically symmetric case, $K_{r}^{r}, K_{\theta}^{\theta}$ and $K_{\varphi}^{\varphi}\left(\propto K_{\theta}^{\theta}\right)$ are the only non-trivial components of $K_{\mu}^{\nu}$. In this simplified context, it is more convenient to introduce the following combinations of these components

$$
X=K_{r}^{r}+K_{\theta}^{\theta}=\frac{\dot{E}^{\phi}-\left(N^{r} E^{\phi}\right)^{\prime}}{N E^{\phi}}, \quad Y=K_{\theta}^{\theta}=\frac{\dot{E}^{x}-N^{r} E^{x \prime}}{2 N E^{x}},
$$

which involve separately the velocities of $E^{\phi}$ and $E^{x}$. This will be very useful for the Hamiltonian analysis. Their covariant form can be given in terms of $\chi_{1}=\square \phi$ and $\chi_{2}=\phi_{\mu \nu} \phi^{\mu \nu}$ only as follows

$$
X=\frac{2}{3} \square \phi+\frac{1}{6} \sqrt{6 \phi_{\mu \nu} \phi^{\mu \nu}-2(\square \phi)^{2}}, \quad Y=\frac{1}{3} \square \phi-\frac{1}{6} \sqrt{6 \phi_{\mu \nu} \phi^{\mu \nu}-2(\square \phi)^{2}} .
$$

Hence, we can view the $\chi_{i}$ variables as functions of only the two variables $X$ and $Y$, and then we can reformulate the general action (4.42) as

$$
S=\int d t d^{3} x N E^{\phi} \sqrt{E^{x}}\left[-\left(2 X Y-Y^{2}\right)+\tilde{L}_{\phi}(X, Y)+\frac{1}{2} R\right],
$$

where $\tilde{L}_{\phi}(X, Y)=L_{\phi}\left(\chi_{1}, \cdots, \chi_{p}\right)$. This expression of the action is much more suitable for a Hamiltonian analysis. First, we compute the momenta conjugated to the variables $E^{x}$ and $E^{\phi}$

$$
\begin{aligned}
& \pi_{x}=\frac{\delta Y}{\delta \dot{E}^{x}} \frac{\delta L}{\delta Y}=\frac{E^{\phi}}{2 \sqrt{E^{x}}}\left(-2(X+Y)+\frac{\partial \tilde{L}_{\phi}}{\partial Y}\right), \\
& \pi_{\phi}=\frac{\delta X}{\delta \dot{E}^{\phi}} \frac{\delta L}{\delta X}=\sqrt{E^{x}}\left(-2 Y+\frac{\partial \tilde{L}_{\phi}}{\partial X}\right)
\end{aligned}
$$


where $L$ denotes the full Lagrangian of the theory. Notice that in the classical limit, where $\tilde{L}_{\phi}(X, Y) \rightarrow 0$, the momenta become "classical" and reduce to the expected form

$$
\pi_{x} \rightarrow \pi_{x}^{c}=-\frac{E^{\phi}}{\sqrt{E^{x}}}(X+Y) \text { and } \pi_{\phi} \rightarrow \pi_{\phi}^{c}=-2 \sqrt{E^{x}} Y .
$$

To compute the Hamiltonian, one has to invert the relations (4.32) and (4.33) in order to express the velocities in terms of the momenta. However, it is not always possible to have an explicit inversion and then to have an explicit form of the Hamiltonian.

Our goal is to find a function $\tilde{L}_{\phi}(X, Y)$ which has the right semi-classical limit and which reproduces the general shape of the quantum correction appearing in the polymer model as shown in (4.11). Since such corrections only affect the angular component of the extrinsic curvature in the polymer model, we focus on $\pi_{\phi}$ in (4.33), and we require that there exists a function $f$ such that

$$
Y=-f\left(\frac{\pi_{\phi}}{\sqrt{E^{x}}}\right)
$$

Remark that such a quantum correction has a natural inbuilt $\bar{\mu}$-scheme through its dependency in $E^{x}$ to keep the covariance. Since the function $f$ does not depend on $X$, we can conclude that the general function $\tilde{L}_{\phi}(X, Y)$ which reproduces the polymer Hamiltonian takes the form:

$$
\tilde{L}_{\phi}(X, Y)=2 X Y+X f^{-1}(-Y)+g(Y),
$$

where $g(Y)$ is a function of $Y$ only which satisfies $g(Y) \rightarrow 0$ in the classical limit. Indeed, this choice of $\tilde{L}_{\phi}(X, Y)$ leads to the action (4.31)

$$
S=\int d t d^{3} x N E^{\phi} \sqrt{E^{x}}\left[Y^{2}+X f^{-1}(Y)+g(Y)+\frac{1}{2} R\right],
$$

whose Hamiltonian (after a few calculations) reads

$$
\begin{aligned}
H & =N\left[2 E^{x} \pi_{x} Y+E^{\phi} \pi_{\phi} X-E^{\phi} \sqrt{E^{x}}\left(Y^{2}+X f^{-1}(Y)+g(Y)+\frac{1}{2} R\right)\right] \\
& =-\frac{N}{\sqrt{E^{x}}}\left[2 E^{x} \pi_{x} f\left(\frac{\pi_{\phi}}{\sqrt{E^{x}}}\right)+E^{\phi}\left(f^{2}\left(\frac{\pi_{\phi}}{\sqrt{E^{x}}}\right)-E^{x} g(Y)\right)+\frac{1}{2} E^{\phi} E^{x} R\right] \\
& =-\beta^{2} \frac{N}{\sqrt{E^{x}}}\left[\frac{2}{\beta} K_{x} E^{x} f\left(\frac{2 \beta K_{\phi}}{\sqrt{E^{x}}}\right)+E^{\phi}\left(\frac{1}{\beta^{2}} f^{2}\left(\frac{2 \beta K_{\phi}}{\sqrt{E^{x}}}\right)-E^{x} g(Y)\right)+\frac{1}{2 \beta^{2}} E^{\phi} E^{x} R\right],
\end{aligned}
$$

where we have used the relations (4.24). Notice that one can express the three-curvature $R$ as in (4.11) but this is not needed here. As a conclusion, one can immediately identify this Hamiltonian with the polymer model Hamiltonian (4.11) with the conditions that

$$
f_{1}\left(E^{x}, K_{\phi}\right)=\frac{1}{\beta^{2}} f^{2}\left(\frac{2 \beta K_{\phi}}{\sqrt{E^{x}}}\right)-E^{x} g(Y) \quad \text { and } \quad f_{2}\left(E^{x}, K_{\phi}\right)=\frac{1}{\beta} f\left(\frac{2 \beta K_{\phi}}{\sqrt{E^{x}}}\right) .
$$

In that way, one obtains an extended theory of mimetic gravity which reproduces the general shape of the holonomy corrections considered in the polymer model, with an additional natural $\bar{\mu}$-scheme. 
Let us now quickly look at the cosmological limit of the previous action. In that case, all the $\chi_{i}$ variables can be expressed in terms of $\square \phi$ only, and we have

$$
X=2 Y=\frac{2}{3} \square \phi .
$$

As a consequence, the mimetic potential takes the very simple form

$$
L_{\phi}=\frac{4}{9}(\square \phi)^{2}+\frac{2}{3} \square \phi f^{-1}\left(\frac{2}{3} \square \phi\right)+g\left(\frac{1}{3} \square \phi\right) .
$$

Having presented our general procedure to derive an effective action reproducing the hamiltonian effective corrections of polymer models, let us briefly summarize our strategy. The general mimetic action we shall investigate in the following, through special examples, is given by

$$
S\left[g_{\mu \nu}, \phi, \lambda\right] \equiv \int d^{4} x \sqrt{-g}\left[\frac{1}{2} \mathcal{R}+2 X Y+X f^{-1}(-Y)+g(Y)+\lambda\left(\phi_{\mu} \phi^{\mu} \pm 1\right)\right]
$$

where the variabes $X$ and $Y$ are given by

$$
X=\frac{2}{3} \square \phi+\frac{1}{6} \sqrt{6 \phi_{\mu \nu} \phi^{\mu \nu}-2(\square \phi)^{2}} \quad Y=\frac{1}{3} \square \phi-\frac{1}{6} \sqrt{6 \phi_{\mu \nu} \phi^{\mu \nu}-2(\square \phi)^{2}} .
$$

and the functions $f^{-1}(-Y)$ and $g(Y)$ are the unknown.

We can now apply our strategy to concrete examples treated in the polymer litterature. An interesting question that we intend to clarify with the next two examples is the following. Considering the two loop regularization proposed so far in spherically symmetric inhomogeneous backgrounds, does their associated effective mimetic actions derived from the procedure outlined above coincide with the Chamseddine-Muhkanov's lagrangian as the cosmological lagrangian? We show below that in order to have the right cosmological limit, the anomaly free condition within the $\bar{\mu}$-scheme are a crucial ingredient.

\subsubsection{A first example: the standard anomaly free sine function corrections}

As a first example, let us consider the standard holonomy corrections given by sine functions which satisfy the anomaly free constraint (4.15):

$$
f_{1}\left(K_{\phi}\right)=\frac{\sin \left(\rho K_{\phi}\right)}{\rho}, \quad f_{2}\left(K_{\phi}\right)=\frac{1}{2} \frac{d f_{1}\left(K_{\phi}\right)}{d K_{\phi}} .
$$

Here the scale $\rho$ is a constant and thus, does not correspond to the $\bar{\mu}$-scheme. As we have seen, when deriving the quantum corrections from the covariant theory, we always obtain a dynamical scale $\rho$ which depend explicitly on the metric. Hence, the corrections (4.44) cannot be reproduced as they stand from our procedure.

A first naive attempt is to generalize the above correction in the $\mu_{0}$ scheme to the $\bar{\mu}$ one, by promoting the scale $\rho$ to a dynamical scale $\tilde{\rho}=2 \rho \beta / \sqrt{E^{x}}$. This generalization is not harmless from the point of view of the anomaly freedom problem. Indeed, when generalizing the corrections function in this way, we should first derive the new associated anomaly free conditions which will be different from (4.44) and then compute the solutions to this conditions (See example 2 below). Here, we proceed to the generalization by hand and show, as expected, the covariant mimetic lagrangian which reproduces these naive $\bar{\mu}$ corrections does not admit 
the cosmological Chamseddine-Mukhanov's lagrangian at its cosmological limit. This first example underlines how the anomaly-free conditions combined with a $\bar{\mu}$-scheme are crucial for the consistency of the model.

After our naive generalization to include by hand a $\bar{\mu}$-scheme, our corrections functions become

$$
f_{1}\left(E^{x}, K_{\phi}\right)=\frac{\sqrt{E^{x}}}{2 \beta \rho} \sin \left(\frac{2 \beta \rho}{\sqrt{E^{x}}} K_{\phi}\right), \quad f_{2}\left(E^{x}, K_{\phi}\right)=\frac{E^{x}}{\beta^{2} \rho^{2}} \sin ^{2}\left(\frac{\beta \rho}{\sqrt{E^{x}}} K_{\phi}\right) .
$$

From the previous construction, we can explicitly derive the mimetic Lagrangian which reproduces such holonomy-like corrections (4.45). Using (4.39), one can first write the angular component of the extrinsic curvature $Y=-K_{\theta}^{\theta}$ as follows

$$
Y=-f\left(\frac{2 \beta K_{\phi}}{\sqrt{E^{x}}}\right)=-\beta f_{2}\left(E^{x}, K_{\phi}\right) .
$$

Then, one easily obtains the expressions of the functions $f$ and $g$ entering in the definition of $\tilde{L}_{\phi}$

$$
f^{-1}(-Y)=\frac{1}{\rho} \arcsin (-2 \rho Y), \quad g(Y)=-Y^{2}+\frac{1}{2 \rho^{2}}\left(1-\sqrt{1-4 \rho^{2} Y^{2}}\right),
$$

which finally leads to the Lagrangian

$$
\tilde{L}_{\phi}=2 X Y-Y^{2}+\frac{X}{\rho} \arcsin (-2 \rho Y)+\frac{1}{2 \rho^{2}}\left(1-\sqrt{1-4 \rho^{2} Y^{2}}\right) .
$$

The symmetry reduction to the cosmological sector leads

$$
L_{\phi}=\frac{1}{3} \square \phi^{2}-\frac{2}{3 \rho} \square \phi \arcsin \left(\frac{2}{3} \rho \square \phi\right)+\frac{1}{2 \rho^{2}}\left(1-\sqrt{1-\frac{4}{9} \rho^{2} \square \phi^{2}}\right) .
$$

As expected, (4.49) does not coincide with the extended mimetic Lagrangian initially proposed by Chamseddine and Mukhanov (2.15) which has been shown to reproduce the LQC dynamics $[8,9]$. Therefore, the naive generalization of the effective corrections (4.44) to (4.45) does admit a covariant mimetic lagrangian albeit with the wrong cosmological limit. The reason is that the corrections (4.45) does not satisfy the rigth anomaly free conditions. We can now turn to the second example which exhibits the right anomaly free conditions.

\subsubsection{A second example: the Tibrewala's effective corrections}

As emphasized above, the corrections functions (4.45) satisfy the anomaly free conditions (4.44) which were derived for corrections functions $f_{1,2}\left(K_{\phi}\right)$. Yet such conditions are not consistent with a $\bar{\mu}$-scheme. Such conditions for corrections functions of the type $f_{1,2}\left(E^{x}, K_{\phi}\right)$ were derived in [7] and read

$$
f_{2}-f_{1} \frac{\partial f_{1}}{\partial K_{\phi}}+2 E^{x} \frac{\partial f_{2}}{\partial E^{x}}=0
$$

Choosing the function $f_{2}$ to be

$$
f_{2}\left(K_{\phi}\right)=\frac{\sqrt{E^{x}} \sin \left(2 \beta \rho K_{\phi} / \sqrt{E^{x}}\right)}{2 \beta \rho}
$$


the second holonomy corrections $f_{1}$ is constrained to be

$$
f_{1}\left(K_{\phi}\right)=\frac{3 E^{x} \sin ^{2}\left(\beta \rho K_{\phi} / \sqrt{E^{x}}\right)}{\beta^{2} \rho^{2}}-2 K_{\phi} \frac{\sqrt{E^{x}} \sin \left(2 \beta \rho K_{\phi} / \sqrt{E^{x}}\right)}{2 \beta \rho}
$$

Following exactly the same strategy as in the previous example, we first find the functions $f$ and $g$ which are given here by

$$
\begin{aligned}
& f^{-1}(-Y)=\frac{1}{\rho} \arcsin (-2 \rho Y), \\
& g(Y)=\frac{3}{2 \rho^{2}}\left(1-\sqrt{1-4 \rho^{2} Y^{2}}\right)-Y^{2}-\frac{\arcsin (2 \rho Y)}{\rho} Y .
\end{aligned}
$$

Then, we deduce immediately the mimetic Lagrangian

$$
\tilde{L}_{\phi}=2 X Y-Y^{2}+\frac{X+Y}{\rho} \arcsin (-2 \rho Y)+\frac{3}{2 \rho^{2}}\left(1-\sqrt{1-4 \rho^{2} Y^{2}}\right) .
$$

Interestingly, the symmetry reduction to the cosmological sector leads to the Lagrangian

$$
L_{\phi}=\frac{1}{3} \square \phi^{2}-\frac{1}{\rho} \square \phi \arcsin \left(\frac{2}{3} \rho \square \phi\right)+\frac{3}{2 \rho^{2}}\left(1-\sqrt{1-\frac{4}{9} \rho^{2} \square \phi^{2}}\right)
$$

which coincides exactly with the Chamseddine-Mukhanov Lagrangian, the one that reproduces the LQC dynamics (2.15). Hence, one can see that the conditions (4.50), which are consistent with a $\bar{\mu}$-scheme, allow to derive a covariant mimetic lagrangian (in the spherically symetric sector) which admits the right cosmological limit, ie the lagrangian reproducing the LQC effective dynamics. The reason behind this result lies in the fact that the corrections (4.52) (derived in [7]) do satisfy consistent anomaly free conditions which take into account, from the start, the existence of a $\bar{\mu}$-scheme.

Yet, let us emphasize a subtelty of our work. The regularized phase space obtained from either implementing the effective corrections at the hamiltonian level as done in [7] and the one obtained from a canonical analysis of the action (4.55) derived in this section, are not the same. The first one has a deformed notion of covariance, the second exhibits an undeformed covariance since it is derived from a covariant action. This suggests that additional ingredients for the loop regularization of [7] to provide an undeformed covariance are encoded in the mimetic covariant action. From this observation, extended mimetic gravity provides indeed an interesting guide to investigate further the covariance issue of the loop regularization performed in polymer black hole and the relation between the two phases spaces should be studied further. We leave this observation for future work.

Finally, we point out that from this investigation, the $\bar{\mu}$-scheme seems to be a necessary ingredient, albeit not sufficient, to have a fully covariant regularization of the phase space of spherically symmetric vacuum gravity and thus, hope to derive effective covariant actions for such polymer models ${ }^{4}$.

\footnotetext{
${ }^{4}$ Note also that, using our procedure, it is possible to derive an effective action for the polymer black hole models which describe the Schwarzschild interior [51, 56], since they are dealing with an anisotropic, albeit homogeneous background. In these models (at least the most recent version), a $\bar{\mu}$-scheme is included, just as standard LQC, and it is straitforward to derive the associated effective mimetic action reproducing their hamiltonian corrections. Yet the true challenge is dealing with inhomogeneous backgrounds, and thus we do not present the effective mimetic action for these polymer black hole interior models.
} 


\section{Conclusion}

In this paper, we start by revisiting the regular black hole interior solution obtained in the context of mimetic gravity [10] from a Hamiltonian perspective. We introduced a suitable parametrization of the static spherically symmetric metric which allowed us to perform a complete Hamiltonian analysis of the theory. We wrote the Hamiltonian equations and we showed that the determinant $\gamma$ of the spatial metric admits an evolution equation very similar to the modified Friedmann equation obtained in effective LQC (3.23). Thus, the black hole has a bounded energy density which prevents the existence of a singularity. Finally, we solved the Hamilton equations in the regime deep inside the black hole and we made a comparison with the standard Schwarzschild solution, showing explicitly that the limiting curvature mechanism indeed resolves the singularity.

Then, motivated by the recent finding that the initial extended mimetic theory reproduces exactly the effective dynamics of LQC, we have investigated whether this result survives in the spherically symmetric sector. We have argued that this cannot be "naively" the case for a very general reason: the spherically symmetric polymer model fails to be covariant, and there is a priori no hope to reproduce this phase space and its effective quantum corrections from a covariant theory such as mimetic gravity. From a more general perspective, the polymer treatment of inhomogeneous backgrounds (cosmological perturbations, spherical symmetry, Gowdy system) is well known to suffer from a lack of covariance. The holonomy corrections usually introduced during the regularization break the Dirac's hypersurface deformation algebra, or at best, lead to its deformation [1-5]. It becomes then crucial to understand either the conceptual and technical consequences of such deformation, either to understand how to cure it. In this work we have presented a new strategy towards the second problem based on mimetic gravity. Since this theory provides a covariant Lagrangian which reproduces exactly the effective LQC dynamics in the cosmological sector, it provides an interesting tool to derive covariant polymer-like Hamiltonian models beyond the cosmological sector to be compared with the existing ones. As such, we view mimetic theory as a guide to derive covariant notion of point-wise holonomy-like corrections in polymer models.

With this issue of covariance in mind, we have presented a general procedure to construct mimetic Lagrangians which admit a Hamiltonian formulation very similar in spirit to existing polymer models of black holes, but which is fully covariant. Then we have applied our procedure to two examples of polymers black hole models: the standard anomaly-free sine corrections model and the Tribrewala's $\bar{\mu}$-scheme corrections model. From the differences between the mimetic and polymer Hamiltonian formulations, one can extract several insights which could be useful when trying to build undeformed covariant polymer black hole models based on the real Ashtekar-Barbero variables. First, the covariant mimetic formulation always contains a $\bar{\mu}$-scheme, i.e. holonomy-like corrections which depend both on $K_{\phi}$ and $E^{x}$ and not only on $K_{\phi}$. As such, the dynamical nature of the polymer scale $\rho$ is a necessary ingredient to maintain the covariance in presence of effective quantum corrections of the polymer type. However, the $\bar{\mu}$-scheme, while necessary, is not sufficient since previous attempts to include it in spherically symmetric models still lead to a deformed notion of covariance [7] (see [14] for a similar conclusion in the context of cosmological perturbations). Therefore additional ingredients are required to maintain the covariance in such effective polymer models. The second difference between the two approaches concerns the object which is polymerized. In the standard polymer approach to the spherically symmetric background, one polymerizes the connection $K_{\phi}$ while the limiting curvature mechanism of mimetic theory suggests that the 
covariance requires to polymerize a more complicated combination of the canonical variables, given by (4.26). The choice of connection is crucial in this procedure, and the AshetkarBarbero connection $K_{\phi}$ might not be the suitable one to consider ${ }^{5}$.

From a more general point of view, our study suggests that the current procedure developed in spherically symmetric polymer models still lacks some crucial ingredients to provide a consistent covariant effective framework. However, let us emphasize again that the polymer models we have considered only involve the minimal loop corrections, i.e. the so called pointwise holonomy corrections. Therefore, it is still possible to improve construction of polymer effective actions by adding the loop corrections. It could well be that the so called triad corrections, associated to the loop regularization of the inverse volume term, conspire with the holonomy corrections to provide an undeformed algebra of first class constraints. This remains to be checked. Moreover, the spherically symmetric sector raises the question of how to concretely deal with the holonomy of the inhomogeneous component of the connection $K_{x}$. Despite some preliminary work on this question, a consistent and tractable implementation of this extended holonomy is still to be understood. Therefore, our results might suggest that polymer models built on solely point-wise holonomy corrections (and with the real AshtekarBarbero variables) could not admit covariant effective action beyond the cosmological sector, and motivate us to look for a generalization of the current regularization used in such polymer models to implement consistently an undeformed notion of covariance (see [77, 78] for some recent proposals in black hole and cosmology in this direction). The recent exact effective black hole metric solutions for polymer black holes obtained in $[12,13]$ show indeed that a generalization of the loop regularization to include a consistent $\bar{\mu}$ scheme is crucial to obtain black hole backgrounds with a consistent semi-classical limit. The present work suggests that extended mimetic gravity could provide an interesting guide towards this goal.

\section{Acknowledgments}

HL thanks the hospitality of the Department of Physics, Florida Atlantic University, where some of the research related to this work was carried out. J.BA would like to thank the Theory Group of APC for its hospitality during his stay in Paris where this project was initiated.

\section{References}

[1] M. Bojowald, S. Brahma and J. D. Reyes, Covariance in models of loop quantum gravity: Spherical symmetry, Phys. Rev. D92 (2015), arxiv:1507.00329

[2] M. Bojowald and S. Brahma, Covariance in models of loop quantum gravity: Gowdy systems, Phys. Rev. D92 (2015) arxiv:1507.00679

[3] M. Bojowald, S. Brahma, U. Buyukcam and F. D'Ambrosio, Hypersurface-deformation algebroids and effective spacetime models, Phys. Rev. D94 (2016) arxiv:1610.08355

[4] M. Bojowald and S. Brahma, Signature change in loop quantum gravity: Two-dimensional midisuperspace models and dilaton gravity, Phys. Rev. D95 (2017) arxiv:1610.08840

[5] M. Bojowald and S. Brahma, Signature change in 2-dimensional black-hole models of loop quantum gravity, arxiv:1610.08850

\footnotetext{
${ }^{5}$ It is already known that this connection does not transform as a true space-time connection under the action of the scalar constraint, and as such, does not provide a good candidate to ensure a fully covariant quantum description when implementing the dynamics. It might be that the same problem emerges already at the effective level in these polymer models.
} 
[6] R. Tibrewala, Inhomogeneities, loop quantum gravity corrections, constraint algebra and general covariance, Class. Quant. Grav 31 (2014) arxiv:1311.1297

[7] R. Tibrewala, Spherically symmetric Einstein-Maxwell theory and loop quantum gravity corrections, Class. Quant. Grav 29 (2012) arxiv:1207.2585

[8] H. Liu, K. Noui, E. Wilson-Ewing and D. Langlois, Effective loop quantum cosmology as a higher-derivative scalar-tensor theory, Class. Quant. Grav 34 (2017) arxiv:1703.10812

[9] N. Bodendorfer, A. Schater and J. Schliemann, On the canonical structure of general relativity with a limiting curvature and its relation to loop quantum gravity, arxiv:1703.10670

[10] A. H. Chamseddine and V. Mukhanov, Nonsingular Black Hole, Eur. Phys. J. C77 (2017) arxiv:1612.05861

[11] R. Gambini and J. Pullin, Loop quantization of the Schwarzschild black hole, Phys. Rev. 110 (2013) arxiv:1302.5265

[12] J. Ben Achour, F. Lamy, H. Liu and K. Noui, Loop quantum deformation of a Schwarzschild black hole: An effective metric, (2018) arxiv:1803.01152

[13] M. Bojowald, S. Brahma and D-H Yeom, Effective line elements and black hole models in canonical (loop) quantum gravity, (2018) arxiv:1803.01119

[14] H. Yu and M. Liu, Anomaly free cosmological perturbations with generalised holonomy correction in loop quantum cosmology, Class. Quant. Grav (2017) arxiv:1711.04991

[15] A. H. Chamseddine and V. Mukhanov, Mimetic Dark Matter, JHEP. 11 (2013) arxiv:1308.5410

[16] M. Chaichian, J. Kluson, M. Oksanen and A. Tureanu, Mimetic dark matter, ghost instability and a mimetic tensor-vector-scalar gravity, JHEP. 12 (2014) arxiv:1404.4008

[17] L. Sebastiani, D. Vagnozzi and R. Myrzakulov, Mimetic gravity: a review of recent developments and applications to cosmology and astrophysics, Adv. High Energy Phys. (2017) arxiv:1612.08661

[18] D. Langlois and K. Noui, Degenerate higher derivative theories beyond Horndeski: evading the Ostrogradski instability, JCAP. 1602 (2016) arxiv:1510.06930

[19] D. Langlois and K. Noui, Hamiltonian analysis of higher derivative scalar-tensor theories, JCAP. 1607 (2016) arxiv:1512.06820

[20] M. Crisostomi, M. Hull, K. Koyama and G. Tasinato, Horndeski: beyond, or not beyond?, JCAP. 1603 (2016) arxiv:1601.04658

[21] M. Crisostomi, K. Koyama and G. Tasinato, Extended Scalar-Tensor Theories of Gravity, JCAP. 1604 (2016) arxiv:1602.03119

[22] J. Ben Achour, D. Langlois and K. Noui, Degenerate higher order scalar-tensor theories beyond Horndeski and disformal transformations, Phys. Rev. D D93 (2016) arxiv:1602.08398

[23] J. Ben Achour, M. Crisostomi, K. Koyama, D. Langlois, K. Noui and G. Tasinato Degenerate higher order scalar-tensor theories beyond Horndeski up to cubic order, JHEP. 12 (2016) arxiv:1608.08135

[24] S. Mukohyama Dark matter as integration constant in Horava-Lifshitz gravity, Phys. Rev. D80 (2019) arxiv:0905.3563

[25] A. H. Chamseddine, V. Mukhanov and V. Alexander, Cosmology with Mimetic Matter, JCAP. 1406 (2014) arxiv:1403.3961

[26] A. H. Chamseddine, V. Mukhanov, Resolving Cosmological Singularities, JCAP. 1703 (2017) arxiv: 1612.05860 
[27] D. Yoshida, J. Qunitin, M. Yamagushi and R. Brandenberger, Cosmological perturbations and stability of nonsingular cosmologies with limiting curvature, Phys. Rev. D96 (2017) arxiv:1704.04184

[28] M. Crisostomi, R. Klein and D. Roest, Higher Derivatives Field Theories: Degeneracy Conditions and Classes, JHEP. 06 (2017) arxiv:1703.01623

[29] A. Ashtekar and P. Singh, Loop Quantum Cosmology: A status report, Class. Quant. Grav. 28 (2011) arxiv:1108.0893

[30] R. C. Helling, Higher curvature counter terms cause the bounce in loop cosmology, (2009) arxiv:0912.3011

[31] E. Alesci and F. Cianfrani, Quantum Reduced Loop Gravity and the foundation of Loop Quantum Cosmology, Int. J. Mod. Phys. D25 (2016) arxiv:1602.05475

[32] E. Alesci and F. Cianfrani, Quantum reduced loop gravity: Universe on a lattice, Phys. Rev. D92 (2015) arxiv:1506.07835

[33] N. Bodendorfer and A. Zipfel, On the relation between reduced quantisation and quantum reduction for spherical symmetry in loop quantum gravity, Class. Quant. Grav. 33 (2016) arxiv:1512.00221

[34] E. R. Livine and M. Martin-Benito, Classical Setting and Effective Dynamics for Spinfoam Cosmology, Class. Quant. Grav. 30 (2013) arxiv:1111.2867

[35] J. Ben Achour, S. Brahma and M. Geiller, New Hamiltonians for loop quantum cosmology with arbitrary spin representations, Phys. Rev. D95 (2017) arxiv:1612.07615

[36] D. M. de Blas, J. Olmedo and T. Pawlowski, Loop quantization of the Gowdy model with local rotational symmetry, (2017) arxiv:1706.05673

[37] M. Bojowald and G. M. Paily, Deformed General Relativity and Effective Actions from Loop Quantum Gravity, Phys. Rev. D86 (2012) arxiv:1112.1899

[38] J. Ben Achour, S. Brahma and A. Marciano, Spherically symmetric sector of self dual Ashtekar gravity coupled to matter: Anomaly-free algebra of constraints with holonomy corrections, Phys. Rev. D96 (2017) arxiv:1608.07314

[39] J. Ben Achour and S. Brahma, Covariance in self dual inhomogeneous effective models of quantum geometry: Spherical symmetry and Gowdy systems, (2017) arxiv:1712.03677

[40] C. Rovelii, Black hole entropy from loop quantum gravity, Phys. Rev. Lett. 77 (1996) arxiv:9603063

[41] A. Ashtekar, J. Baez, A. Corichi and K. Krasnov, Quantum geometry and black hole entropy, Phys. Rev. Lett. 80 (1998) arxiv:9710007

[42] E. Frodden, M. Geiller, K. Noui and A. Perez, Black Hole Entropy from complex Ashtekar variables, EPL. 107 (2014) arxiv:1212.4060

[43] J. Ben Achour, A. Mouchet and K. Noui, Analytic Continuation of Black Hole Entropy in Loop Quantum Gravity, JHEP. 06 (2015) arxiv:1406.6021

[44] J. Ben Achour, K. Noui and A. Perez, Analytic continuation of the rotating black hole state counting, JHEP. 08 (2016) arxiv:1607.02380

[45] J. Ben Achour and K. Noui, Analytic continuation of real Loop Quantum Gravity : Lessons from black hole thermodynamics, Proceedings, 14th International Symposium Frontiers of Fundamental Physics (FFP14): Marseille, France, July 15-18, 2014. FFP14 (2016) arxiv:1501.05523

[46] M. Geiller and K. Noui, Near-Horizon Radiation and Self-Dual Loop Quantum Gravity, Gen. Rel. Grav. 105 (2014) arxiv:1212.5064 
[47] M. Geiller and K. Noui, A note on the Holst action, the time gauge, and the Barbero-Immirzi parameter, Gen. Rel. Grav. 45 (2013) arxiv:1402.4138

[48] J. Ben Achour, M. Geiller, K. Noui and C. Yu, Testing the role of the Barbero-Immirzi parameter and the choice of connection in Loop Quantum Gravity, Phys. Rev. D91 (2015) arxiv: 1306.3241

[49] J. Ben Achour, M. Geiller, K. Noui and C. Yu, Spectra of geometric operators in three-dimensional loop quantum gravity: From discrete to continuous, Phys. Rev. D89 (2014) arxiv: 1306.3246

[50] A. Ashtekar and M. Bojowald, Quantum geometry and the Schwarzschild singularity, Class. Quant. Grav. 23 (2006) arxiv:0509075

[51] A. Ashtekar and M. Bojowald, Quantum geometry and the Schwarzschild singularity, Class. Quant. Grav. 23 (2006) arxiv:0509075

[52] C. G. Boehmer and K. Vandersloot, Loop Quantum Dynamics of the Schwarzschild Interior, Phys. Rev. D76 (2007) arxiv:0709.2129

[53] L. Modesto, The Kantowski-Sachs space-time in loop quantum gravity, Int. J. Theor. Phys. 45 (2006) arxiv:0411032

[54] L. Modesto, Black hole interior from loop quantum gravity, Adv. High Energy Phys. (2008) arxiv:0611043

[55] M. Campiglia, R. Gambini and J. Pullin, Loop quantization of spherically symmetric midi-superspaces : The Interior problem, AIP Conf. Proc. 977 (2008) arxiv:0712.0817

[56] A. Corichi and P. Singh, Loop quantization of the Schwarzschild interior revisited, Class. Quant. Grav. 33 (2006) arxiv:1506.08015

[57] R. Gambini and J. Pullin, Black holes in loop quantum gravity: The Complete space-time, Phys. Rev. Lett. 101 (2008) arxiv:0805.1187

[58] R. Gambini, J. Olemdo and J. Pullin, Quantum black holes in Loop Quantum Gravity, Class. Quant. Grav. 31 (2014) arxiv:1310.5996

[59] C. Rovelli and F. Vidotto, Planck stars, Int. J. Mod. Phys. D23 (2014) arxiv:1401.6562

[60] A. Barrau and C. Rovelli, Planck star phenomenology, Phys. Lett. B739 (2014) arxiv:1404.5821

[61] M. Christodoulou, C. Rovelli, S. Speziale and I. Vilensky, Planck star tunneling time: An astrophysically relevant observable from background-free quantum gravity, Phys. Rev. D94 (2016) arxiv:1605.05268

[62] T. De Lorenzo, C. Pacillo, C. Rovelli and S. Speziale, On the Effective Metric of a Planck Star, Gen. Rel. Grav. 47 (2015) arxiv:1412.6015

[63] K. Takahashi and T. Kobayashi, Extended mimetic gravity: Hamiltonian analysis and gradient instabilities, (2017) arxiv:1708.02951

[64] D. Langlois, M. Marcella, K. Noui and F. Vernizzi, Effective Description of Higher-Order Scalar-Tensor Theories, JCAP 1705 (2017) arxiv:1703.03797

[65] D. Langlois, M. Mancarella, K. Noui, and F. Vernizzi, "Mimetic gravity as DHOST theories," arxiv:1802.03394

[66] M. A. Gorji, M. Hosseini, A. Seyed and H. Firouzjahi, Higher Derivative Mimetic Gravity, (2017) arxiv:1709.09988

[67] E. Babichev, C. Charmoussis and A. Lehebel, Black holes and stars in Horndeski theory, Class. Quant. Grav. 33 (2016) arxiv:1604.06402

[68] E. Babichev, C. Charmoussis and A. Lehebel, Black holes in a cubic Galileon universe, JCAP 1609 (2016) arxiv:1605.07438 
[69] M. Bojowald, Spherically symmetric quantum geometry: States and basic operators, Class. Quant. Grav. 21 (2004) arxiv:0407017

[70] T. Cailletaux, J. Mielczarek, A. Barrau and J. Grain, Anomaly-free scalar perturbations with holonomy corrections in loop quantum cosmology, Class. Quant. Grav. 29 (2012) arxiv:1111.3535

[71] J. Grain, The perturbed universe in the deformed algebra approach of Loop Quantum Cosmology, Int. J. Mod. Phys. D25 (2016) arxiv:1606.03271

[72] M. Bojowald and J. Mielczarek, Some implications of signature-change in cosmological models of loop quantum gravity, JCAP. 1508 (2015) arxiv:1503.09154

[73] J. Mielczarek, L. Linsefors and A. Barrau, Silent initial conditions for cosmological perturbations with a change of space-time signature, (2014) arxiv:1411.0272

[74] A. Barrau and J. Grain, Cosmology without time: What to do with a possible signature change from quantum gravitational origin?, (2016) arxiv:1607.07589

[75] M. Bojowald, Information loss, made worse by quantum gravity?, Front.in Phys. 3 (2015) arxiv:1409.3157

[76] J. Ben Achour, S. Brahma, J. Grain and A. Marciano, A new look at scalar perturbations in loop quantum cosmology: (un)deformed algebra approach using self dual variables, (2016) arxiv:1610.07467

[77] M. Campiglia, R. Gambini, J. Olmedo and J. Pullin, Quantum self-gravitating collapsing matter in a quantum geometry, Class. Quant. Grav. 33 (2016) arxiv:1601.05688

[78] J. Ben Achour and E. R. Livine, Thiemann complexifier in classical and quantum FLRW cosmology, Phys. Rev. D96 (2017) arxiv:1705.03772 